\title{
Dynamic Characteristics of Four Systems of Difference Equations with Higher Order
}

\author{
Abdul Qadeer Khan $\mathbb{D}^{1},{ }^{1}$ Shahid Mahmood Qureshi, ${ }^{1}$ and Imtiaz Ahmed ${ }^{2}{ }^{2}$ \\ ${ }^{1}$ Department of Mathematics, University of Azad Jammu and Kashmir, Muzaffarabad 13100, Pakistan \\ ${ }^{2}$ Department of Mathematics, Mirpur University of Science and Technology (MUST), Mirpur 10250 AJK, Pakistan
}

Correspondence should be addressed to Abdul Qadeer Khan; abdulqadeerkhan1@gmail.com

Received 17 August 2021; Revised 18 October 2021; Accepted 13 November 2021; Published 13 December 2021

Academic Editor: Mohammad Mirzazadeh

Copyright (c) 2021 Abdul Qadeer Khan et al. This is an open access article distributed under the Creative Commons Attribution License, which permits unrestricted use, distribution, and reproduction in any medium, provided the original work is properly cited.

In this paper, we explore the global dynamical characteristics, boundedness, and rate of convergence of certain higher-order discrete systems of difference equations. More precisely, it is proved that for all involved respective parameters, discrete systems have a trivial fixed point. We have studied local and global dynamical characteristics at trivial fixed point and proved that trivial fixed point of the discrete systems is globally stable under respective definite parametric conditions. We have also studied boundedness and rate of convergence for under consideration discrete systems. Finally, theoretical results are confirmed numerically. Our findings in this paper are considerably extended and improve existing results in the literature.

\section{Introduction}

1.1. Motivation and Literature Review. No one can deny the significance of difference equations these days. These equation models not only are the discrete physical phenomenon but also are the integral part of numerical schemes used to solve differential equations. These equations are widely applied in many branches of scientific field. Difference equations describe the phenomenon of discrete dynamical systems and have applications in various branches of science such as statistical problems, resource management, neural networks, ecology, economics, queuing problems, number theory, sociology, physics, engineering, psychology, quanta in radiation, genetics in biology, combinatorial analysis, probability theory, geometry, population dynamics, electrical networks, stochastic time series, and queuing problems $[1,2]$. Studying the global characteristics of higher-order nonlinear difference equations or systems of difference equations is a difficult but rewarding task. These findings pave the way for the construction of a basic theory of higherorder difference equations. Recently, many mathematicians have explored the dynamics of difference equation along their system. For instance, Oğul et al. [3] investigated the dynamics of the following higher-order system:

$$
x_{n+1}=\frac{x_{n-15}}{ \pm 1 \pm x_{n-3} x_{n-7} x_{n-11} x_{n-15}} .
$$

Kulenović et al. [4] investigated the dynamic behavior of the difference equation:

$$
\mathrm{x}_{n+1}=\frac{\alpha x_{n}+\beta x_{n-1}}{A+x_{n-1}} .
$$

Zhang et al. [5] investigated the dynamic behavior of the difference equation system:

$$
\begin{aligned}
& x_{n+1}=A+\frac{x_{n}}{\sum_{i=1}^{k} y_{n-i}}, \\
& y_{n+1}=B+\frac{y_{n}}{\sum_{i=1}^{k} x_{n-i}} .
\end{aligned}
$$

Kalabusisić et al. [6] investigated the dynamic behavior of the difference equation system:

$$
\begin{aligned}
& x_{n+1}=\frac{\alpha_{1}+\beta_{1} x_{n}}{y_{n}}, \\
& y_{n+1}=\frac{\alpha_{2}+\gamma_{2} y_{n}}{A_{2}+x_{n}} .
\end{aligned}
$$


Kalabusisic et al. [7] investigated the dynamic behavior of the difference equation system:

$$
\begin{gathered}
x_{n+1}=\frac{\alpha_{1}+\beta_{1} x_{n}}{A_{1}+y_{n}}, \\
y_{n+1}=\frac{\gamma_{2} y_{n}}{A_{2}+B_{2} x_{n}+y_{n}} .
\end{gathered}
$$

Kalabusić et al. [8] investigated the dynamic behavior of the difference equation system:

$$
\begin{aligned}
& x_{n+1}=\frac{\beta_{1} x_{n}}{B_{1} x_{n}+y_{n}}, \\
& y_{n+1}=\frac{\alpha_{2}+\gamma_{2} y_{n}}{A_{2}+x_{n}} .
\end{aligned}
$$

Garić-Demirović et al. [9] investigated the dynamic behavior of four distinct difference equation systems. Elsayed [10,11] studied solutions form of difference equations and their systems. Further, Khan and Qureshi [12] studied the dynamic behavior of a competitive system. DeVault et al. [13] investigated the dynamic behavior of the difference equation:

$$
x_{n+1}=\frac{A}{x_{n}}+\frac{1}{x_{n-2}} .
$$

Abu-Saris and DeVault [14] studied global attractivity of the difference equation:

$$
x_{n+1}=A+\frac{x_{n}}{x_{n-k}} .
$$

On the other hand, in recent years, many works have been published that discussed dynamic behavior of difference equation along their systems [15-20]. In continuation of existing study, it is important to mention that dynamical characteristics of the following difference equation have been investigated by Bajo and Liz [21]:

$$
x_{n+1}=\frac{x_{n-1}}{\alpha_{1}+\alpha_{2} \prod_{i=0}^{1} x_{n-i}},
$$

where $\alpha_{\ell}(\ell=1,2)$ and $x_{-\ell}(\ell=1,0)$ are real constants. By extending the work of [21], Zhang et al. [22] have explored the dynamical properties of the system:

$$
\begin{aligned}
& x_{n+1}=\frac{x_{n-2}}{\alpha_{2}+\prod_{i=0}^{2} y_{n-i}}, \\
& y_{n+1}=\frac{y_{n-2}}{\alpha_{1}+\prod_{i=0}^{2} x_{n-i}},
\end{aligned}
$$

where $\alpha_{\ell}(\ell=1,2)$ and $x_{-\ell}, y_{-\ell}(\ell=2,1,0)$ are real constants.

1.2. Objective, Contributions, and Novelties. Motivated from the aforementioned studies, the objective of the present study is to investigate the behavior of certain rational systems by extending the work done by $[21,22]$ :

$$
\begin{aligned}
& x_{n+1}=\frac{\alpha_{10} y_{n-k}}{\alpha_{11}+\alpha_{12} \prod_{i=0}^{k} z_{n-i}}, \\
& y_{n+1}=\frac{\alpha_{13} z_{n-k}}{\alpha_{14}+\alpha_{15} \prod_{i=0}^{k} x_{n-i}} \text {, } \\
& z_{n+1}=\frac{\alpha_{16} x_{n-k}}{\alpha_{17}+\alpha_{18} \prod_{i=0}^{k} y_{n-i}} \text {, } \\
& x_{n+1}=\frac{\alpha_{19} z_{n-k}}{\alpha_{20}+\alpha_{21} \prod_{i=0}^{k} x_{n-i}} \text {, } \\
& y_{n+1}=\frac{\alpha_{22} x_{n-k}}{\alpha_{23}+\alpha_{24} \prod_{i=0}^{k} y_{n-i}} \text {, } \\
& z_{n+1}=\frac{\alpha_{25} y_{n-k}}{\alpha_{26}+\alpha_{27} \prod_{i=0}^{k} z_{n-i}} \text {, } \\
& x_{n+1}=\frac{\alpha_{28} y_{n-k}}{\alpha_{29}+\alpha_{30} \prod_{i=0}^{k} y_{n-i}} \text {, } \\
& y_{n+1}=\frac{\alpha_{31} z_{n-k}}{\alpha_{32}+\alpha_{33} \prod_{i=0}^{k} z_{n-i}} \text {, } \\
& z_{n+1}=\frac{\alpha_{34} x_{n-k}}{\alpha_{35}+\alpha_{36} \prod_{i=0}^{k} x_{n-i}} \text {, } \\
& x_{n+1}=\frac{\alpha_{37} z_{n-k}}{\alpha_{38}+\alpha_{39} \prod_{i=0}^{k} z_{n-i}} \text {, } \\
& y_{n+1}=\frac{\alpha_{40} x_{n-k}}{\alpha_{41}+\alpha_{42} \prod_{i=0}^{k} x_{n-i}} \text {, } \\
& z_{n+1}=\frac{\alpha_{43} y_{n-k}}{\alpha_{44}+\alpha_{45} \prod_{i=0}^{k} y_{n-i}} \text {, }
\end{aligned}
$$

where $\alpha_{\ell}(\ell=10, \cdots, 45)$ and $x_{-\ell}, y_{-\ell}, z_{-\ell}(\ell=-k,-k+1, \cdots, 1,0$ ) are real constants. More precisely, our main finding in this paper includes the following:

(i) Exploration of trivial fixed point of discrete systems (11)-(14)

(ii) Construction of the corresponding linearized system

(iii) Investigation of global dynamics by stability theory

(iv) Study of boundedness of positive solution and convergence rate of discrete systems (11)-(14)

(v) Validation of obtained results numerically

1.3. Paper Structure. The rest of the paper is structured as follows: In the subsequent section, we explore trivial fixed point and linearized form of discrete systems (11)-(14). Local dynamical characteristics of systems (11)-(14) are investigated in Section 3 while the boundedness for (11)-(14) is explored in Section 4. In Section 5, global dynamics is investigated. Section 6 includes the investigation of rate of converges for said discrete systems. Obtained results are numerically confirmed in Section 7. The conclusion and future work are given in Section 8. 


\section{Linearized Form and Trivial Fixed Point of Systems (11)-(14)}

Linearized form and trivial fixed points of discrete systems (11)-(14) are studied in this section.

2.1. Fixed Point. Obviously, $P_{0}=(0,0,0)$ is the trivial fixed point of discrete systems (11)-(14). Now, in the rest of the section, linearized form for discrete systems (11)-(14) is explored.

2.2. Linearized Form of Discrete System (11). The map for the linearized form of discrete system (11) is

$$
\begin{gathered}
\left(\beta^{(11)}, \beta_{n}^{(11)}, \cdots, \beta_{n-k}^{(11)}, \gamma^{(11)}, \gamma_{n}^{(11)}, \cdots, \gamma_{n-k}^{(11)}, \delta^{(11)}, \delta_{n}^{(11)}, \cdots, \delta_{n-k}^{(11)}\right) \mapsto \widehat{\Gamma} \\
=\left(x_{n+1}, x_{n}, \cdots, x_{n-k+1}, y_{n+1}, y_{n}, \cdots, y_{n-k+1}, z_{n+1}, z_{n}, \cdots, z_{n-k+1}\right),
\end{gathered}
$$

2.3. Linearized Form of Discrete System (12). Linearized form of discrete system (12) at $\Lambda$ under the map: where

$$
\begin{aligned}
& \beta^{(11)}=\frac{\alpha_{10} y_{n-k}}{\alpha_{11}+\alpha_{12} \prod_{i=0}^{k} z_{n-i}}, \beta_{n}^{(11)}=x_{n}, \cdots, \beta_{n-k}^{(11)}=x_{n-k+1}, \\
& \gamma^{(11)}=\frac{\alpha_{13} z_{n-k}}{\alpha_{14}+\alpha_{15} \prod_{i=0}^{k} x_{n-i}}, \gamma_{n}^{(11)}=y_{n}, \cdots, \gamma_{n-k}^{(11)}=y_{n-k+1}, \\
& \delta^{(11)}=\frac{\alpha_{16} x_{n-k}}{\alpha_{17}+\alpha_{18} \prod_{i=0}^{k} y_{n-i}}, \delta_{n}^{(11)}=z_{n}, \cdots, \delta_{n-k}^{(11)}=z_{n-k+1} .
\end{aligned}
$$

The linearized form of (11) at $\Lambda$ under (15) is

$$
\Gamma_{n+1} \wedge^{(11)}=\left.J\right|_{\Lambda} \Gamma_{n} \wedge^{(11)},
$$

$$
\begin{aligned}
& \text { where } \quad \Gamma_{n} \wedge^{(11)}= \\
& \left(x_{n}, x_{n-1}, \cdots, x_{n-k}, y_{n}, y_{n-1}, \cdots, y_{n-k}, z_{n}, z_{n-1}, \cdots, z_{n-k}\right)^{T} \text {, }
\end{aligned}
$$$$
\text { is }
$$

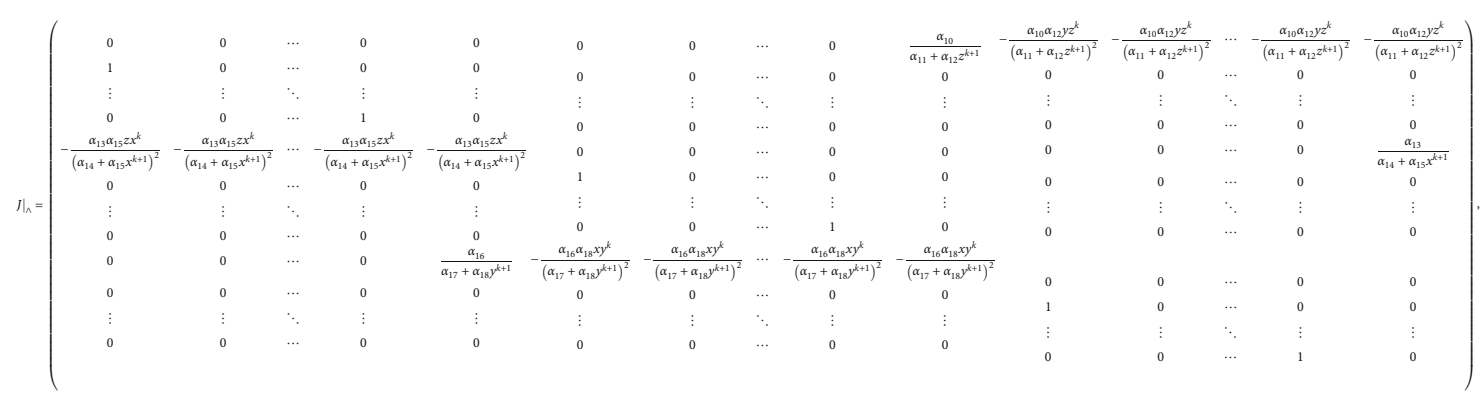

$$
\Lambda=(x, y, z) .
$$

$$
\left(\beta^{(12)}, \beta_{n}^{(12)}, \cdots, \beta_{n-k}^{(12)}, \gamma^{(12)}, \gamma_{n}^{(12)}, \cdots, \gamma_{n-k}^{(12)}, \delta^{(12)}, \delta_{n}^{(12)}, \cdots, \delta_{n-k}^{(12)}\right) \mapsto \widehat{\Gamma}, \quad \Gamma_{n+1} \wedge^{(12)}=\left.J\right|_{\Lambda} \Gamma_{n} \wedge^{(12)}
$$

(20) where

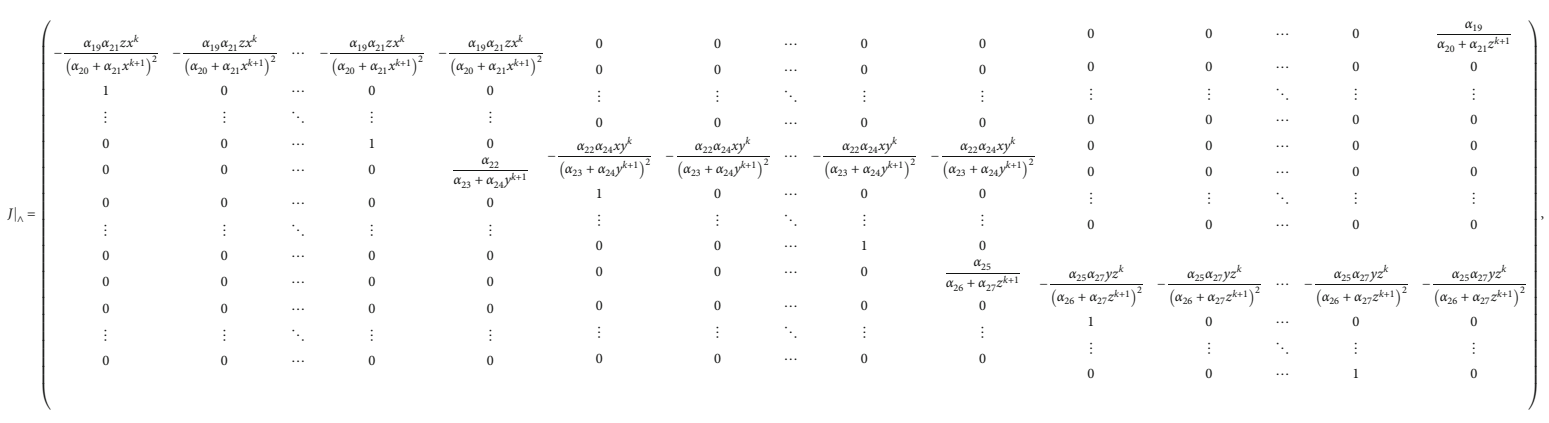


and

$$
\begin{aligned}
& \beta^{(12)}=\frac{\alpha_{19} z_{n-k}}{\alpha_{20}+\alpha_{21} \prod_{i=0}^{k} x_{n-i}}, \beta_{n}^{(12)}=x_{n}, \cdots, \beta_{n-k}^{(12)}=x_{n-k+1}, \\
& \gamma^{(12)}=\frac{\alpha_{22} x_{n-k}}{\alpha_{23}+\alpha_{24} \prod_{i=0}^{k} y_{n-i}}, \gamma_{n}^{(12)}=y_{n}, \cdots, \gamma_{n-k}^{(12)}=y_{n-k+1}, \\
& \delta^{(12)}=\frac{\alpha_{25} y_{n-k}}{\alpha_{26}+\alpha_{27} \prod_{i=0}^{k} z_{n-i}}, \delta_{n}^{(12)}=z_{n}, \cdots, \delta_{n-k}^{(12)}=z_{n-k+1} .
\end{aligned}
$$

of discrete system (13) at $\Lambda$ under the map:

$$
\left(\beta^{(13)}, \beta_{n}^{(13)}, \cdots, \beta_{n-k}^{(13)}, \gamma^{(13)}, \gamma_{n}^{(13)}, \cdots, \gamma_{n-k}^{(13)}, \delta^{(13)}, \delta_{n}^{(13)}, \cdots, \delta_{n-k}^{(13)}\right) \mapsto \widehat{\Gamma}
$$

is

$$
\Gamma_{n+1} \wedge^{(13)}=\left.J\right|_{\Lambda} \Gamma_{n} \wedge^{(13)},
$$

where

\subsection{Linearized Form of Discrete System (13). Linearized form}

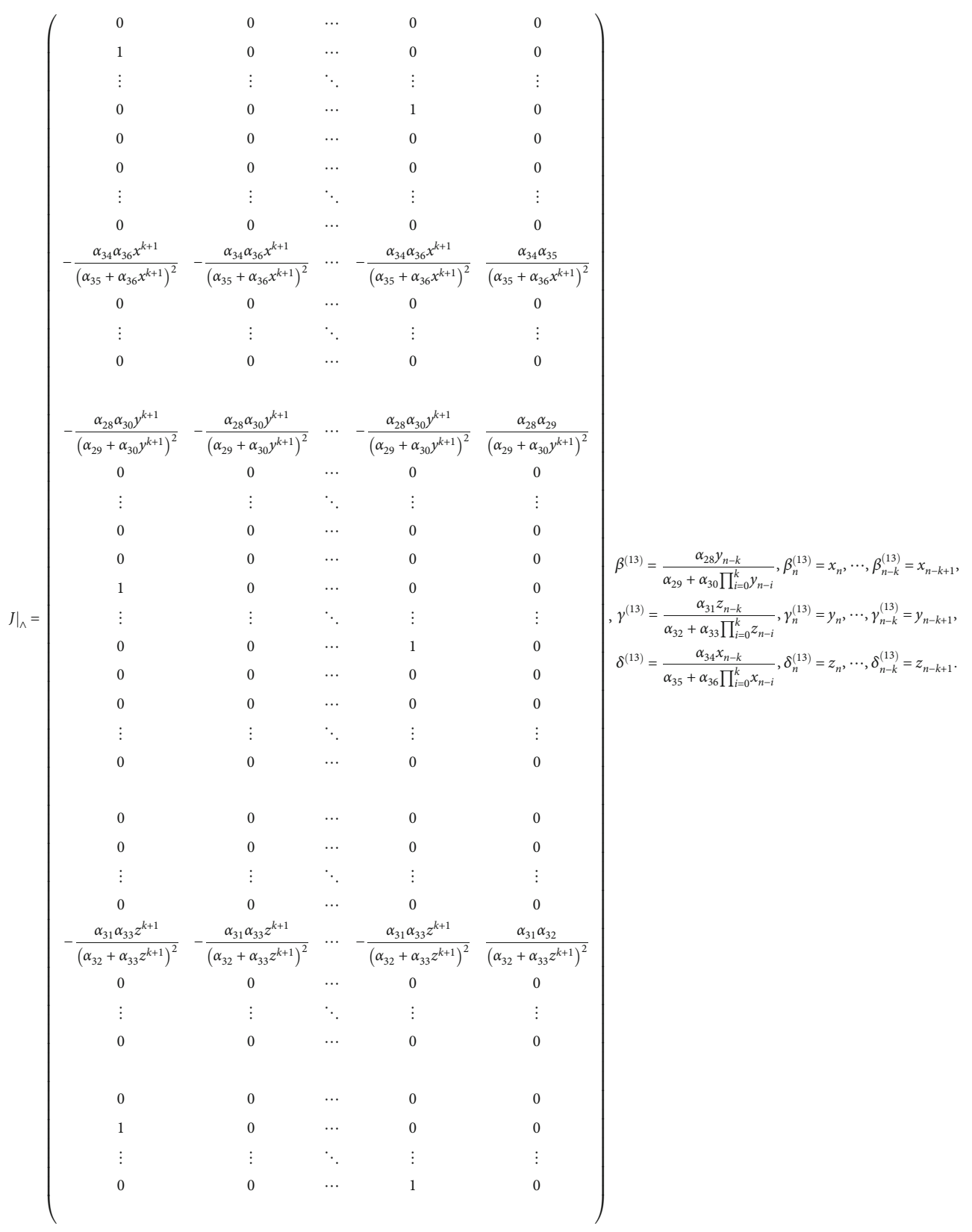


2.5. Linearized Form of Discrete System (14). Linearized form of discrete system (14) at $\Lambda$ under the map:

$$
\left(\beta^{(14)}, \beta_{n}^{(14)}, \cdots, \beta_{n-k}^{(14)}, \gamma^{(14)}, \gamma_{n}^{(14)}, \cdots, \gamma_{n-k}^{(14)}, \delta^{(14)}, \delta_{n}^{(14)}, \cdots, \delta_{n-k}^{(14)}\right) \mapsto \widehat{\Gamma}
$$

is

$$
\Gamma_{n+1} \wedge^{(14)}=\left.J\right|_{\Lambda} \Gamma_{n} \wedge^{(14)}
$$

where

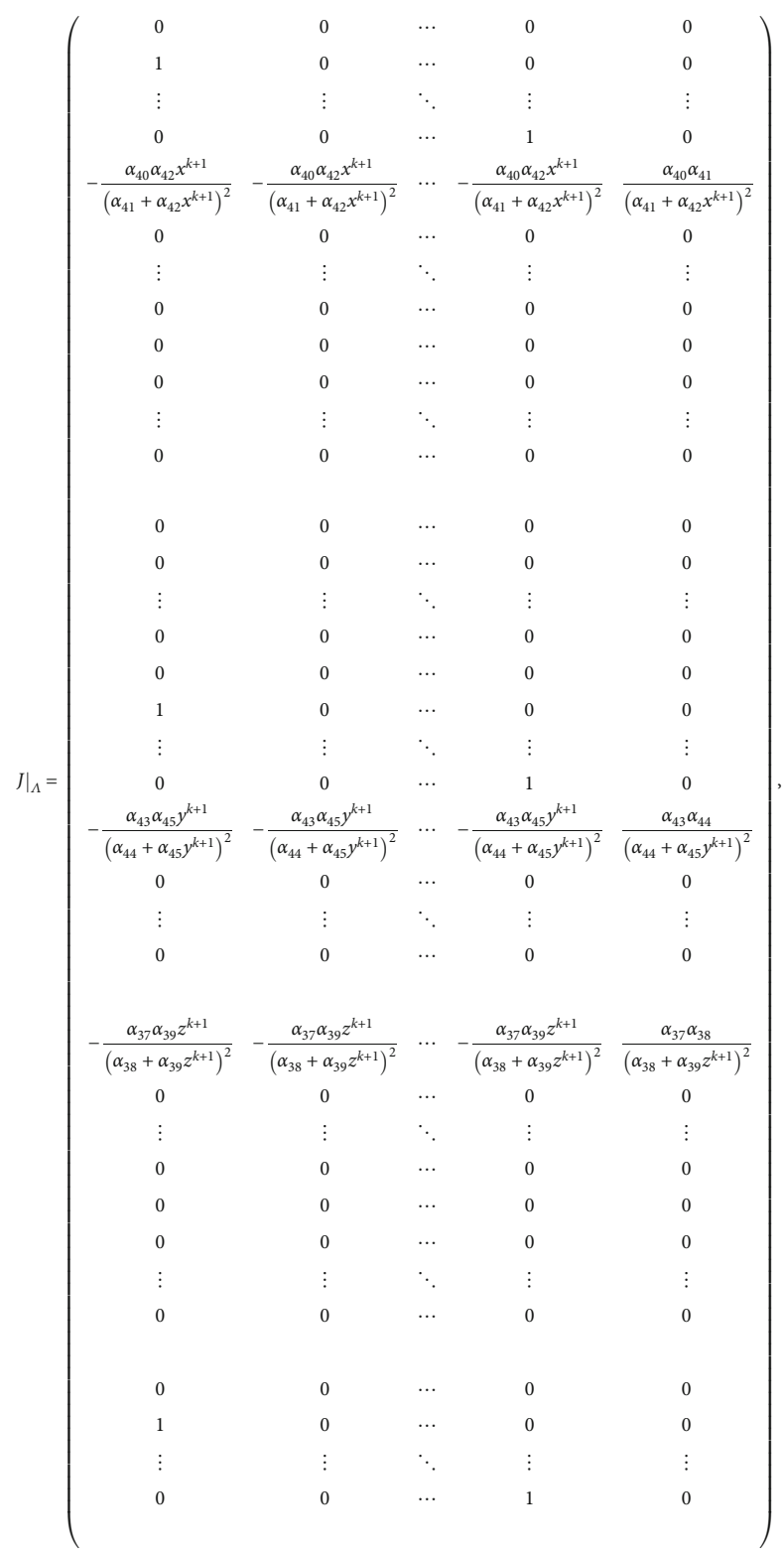

$$
\begin{aligned}
& \beta^{(14)}=\frac{\alpha_{37} z_{n-k}}{\alpha_{38}+\alpha_{39} \prod_{i=0}^{k} z_{n-i}}, \beta_{n}^{(14)}=x_{n}, \cdots, \beta_{n-k}^{(14)}=x_{n-k+1}, \\
& \gamma^{(14)}=\frac{\alpha_{40} x_{n-k}}{\alpha_{41}+\alpha_{42} \prod_{i=0}^{k} x_{n-i}}, \gamma_{n}^{(14)}=y_{n}, \cdots, \gamma_{n-k}^{(14)}=y_{n-k+1}, \\
& \delta^{(14)}=\frac{\alpha_{43} y_{n-k}}{\alpha_{43}+\alpha_{45} \prod_{i=0}^{k} y_{n-i}}, \delta_{n}^{(14)}=z_{n}, \cdots, \delta_{n-k}^{(14)}=z_{n-k+1} .
\end{aligned}
$$

\section{Local Dynamical Characteristics of Discrete Systems (11)-(14)}

Hereafter by Theorem 1.1 of [16], local dynamical characteristics about $P_{0}$ of discrete systems (11)-(14) is explored.

3.1. Local Dynamical Characteristics of Discrete System (11)

Theorem 1. $P_{0}$ of discrete system (11) is a sink if

$$
\begin{aligned}
& \frac{\alpha_{10}}{\alpha_{11}}<1 \\
& \frac{\alpha_{13}}{\alpha_{14}}<1 \\
& \frac{\alpha_{16}}{\alpha_{17}}<1
\end{aligned}
$$

Proof. At $P_{0}$, (17) becomes

$$
\Gamma_{n+1} \wedge^{(11)}=\left.J\right|_{P_{0}} \Gamma_{n} \wedge^{(11)}
$$

From (18), one has

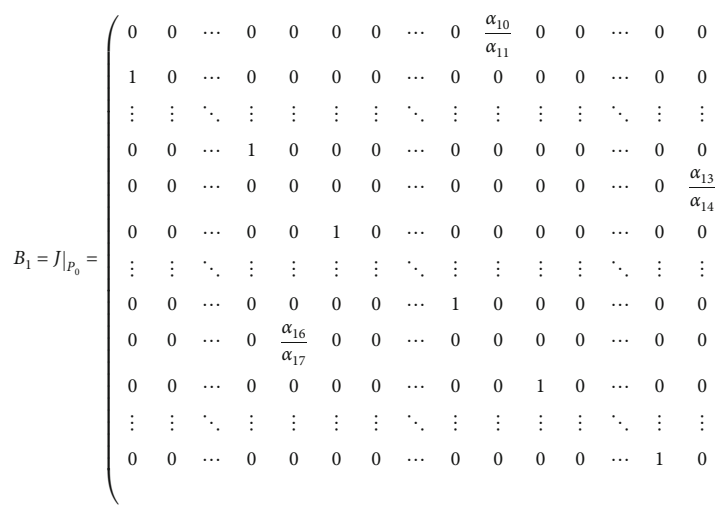

Now, if $\sigma_{1}, \sigma_{2}, \sigma_{3}, \cdots, \sigma_{k+1}, \cdots, \sigma_{2 k+1}, \cdots, \sigma_{3 k+3}$ represent characteristic roots of $P_{0}$ and diagonal matrix: $\widehat{\Omega}=\operatorname{diag}$ $\left(\varrho_{1}, \varrho_{2}, \cdots, \varrho_{k+1}, \cdots, \varrho_{2 k+1}, \cdots, \varrho_{3 k+3}\right)$ where

$$
\begin{gathered}
\varrho_{1}=\varrho_{k+2}=\varrho_{2 k+3}=1, \\
\varrho_{1+\varsigma}=\varrho_{k+2+\varsigma}=\varrho_{2 k+3+\varsigma}=1-\varsigma \varepsilon, 1 \leq \varsigma \leq k \text { where } 0<\varepsilon<1,
\end{gathered}
$$


$0<\varepsilon<\min \left\{\frac{1}{k}\left(1-\frac{\alpha_{10}}{\alpha_{11}}\right), \frac{1}{k}\left(1-\frac{\alpha_{13}}{\alpha_{14}}\right), \frac{1}{k}\left(1-\frac{\alpha_{16}}{\alpha_{17}}\right)\right\}$.

Now,

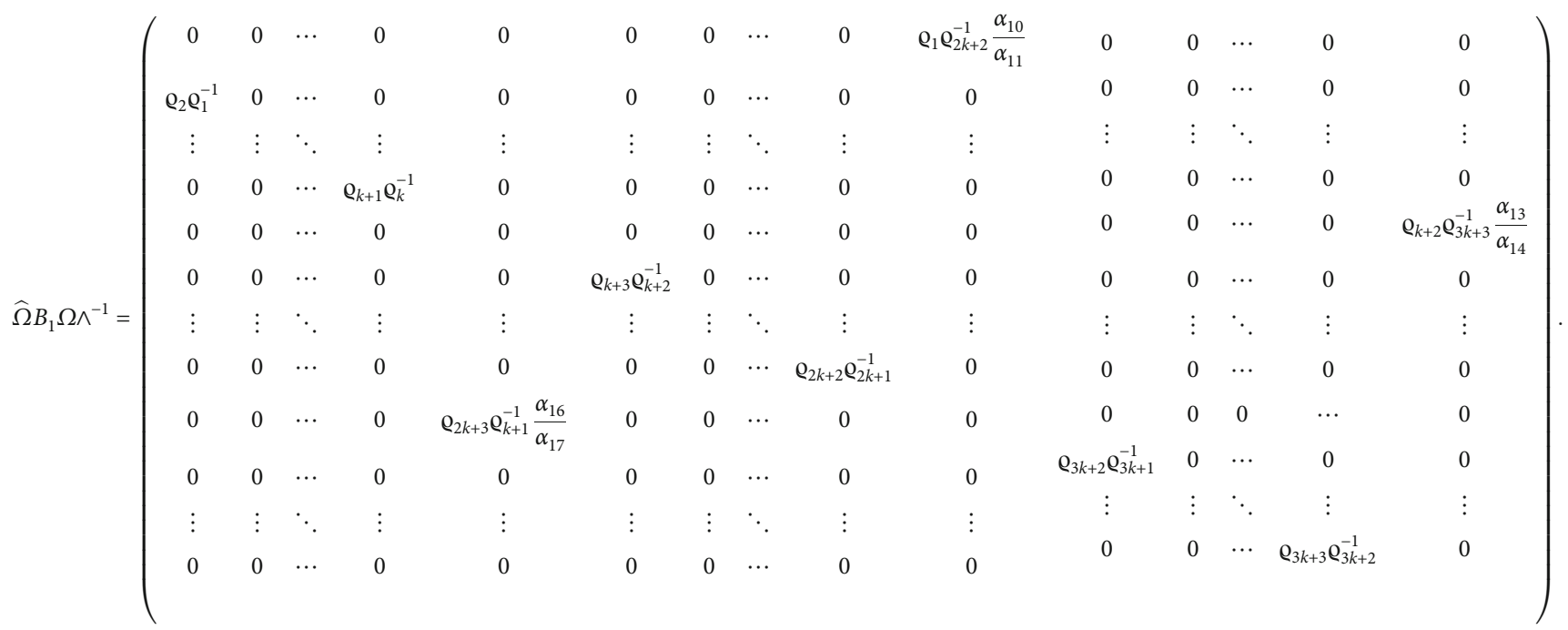

So,

$$
\begin{gathered}
0<\mathrm{Q}_{k+1}<\cdots<\mathrm{Q}_{2}<\mathrm{Q}_{1}, \\
0<\mathrm{Q}_{2 k+2}<\cdots<\mathrm{Q}_{k+2}, \\
0<\mathrm{Q}_{3 k+3}<\cdots<\mathrm{Q}_{2 k+3} .
\end{gathered}
$$

From (36), one obtains

$$
\begin{gathered}
\mathrm{\varrho}_{2} \mathrm{\varrho}_{1}^{-1}<1, \cdots, \varrho_{k+1} \varrho_{k}^{-1}<1, \\
\varrho_{k+3} \varrho_{k+2}^{-1}<1, \cdots, \varrho_{2 k+2} \varrho_{2 k+1}^{-1}<1, \\
\varrho_{2 k+4} \varrho_{2 k+3}^{-1}<1, \cdots, \varrho_{3 k+3} \varrho_{3 k+2}^{-1}<1 .
\end{gathered}
$$

From (33) and (34), one gets

$$
\begin{gathered}
\mathrm{\varrho}_{1} \mathrm{\varrho}_{2 k+2}^{-1} \frac{\alpha_{10}}{\alpha_{11}}=\mathrm{\varrho}_{2 k+2}^{-1} \frac{\alpha_{10}}{\alpha_{11}}=\frac{\alpha_{10}}{\alpha_{11}} \frac{1}{1-k \varepsilon}<1, \\
\mathrm{\varrho}_{k+2} \mathrm{\varrho}_{3 k+3}^{-1} \frac{\alpha_{13}}{\alpha_{14}}=\mathrm{\varrho}_{3 k+3}^{-1} \frac{\alpha_{13}}{\alpha_{14}}=\frac{\alpha_{13}}{\alpha_{14}} \frac{1}{1-k \varepsilon}<1, \\
\mathrm{\varrho}_{2 k+3} \mathrm{\varrho}_{k+1}^{-1} \frac{\alpha_{16}}{\alpha_{17}}=\mathrm{\varrho}_{k+1}^{-1} \frac{\alpha_{16}}{\alpha_{17}}=\frac{\alpha_{16}}{\alpha_{17}} \frac{1}{1-k \varepsilon}<1 .
\end{gathered}
$$

Finally, from (37) and (38), one gets

$$
\begin{aligned}
\max _{1 \leq v \leq 3 k+3}\left|\rho_{v}\right|= & \left\|\widehat{\Omega} B_{1} \Omega \wedge^{-1}\right\|=\max \left\{\mathrm{\varrho}_{1} \mathrm{\varrho}_{2 k+2}^{-1} \frac{\alpha_{10}}{\alpha_{11}}, \varrho_{2} \mathrm{\varrho}_{1}^{-1}, \cdots,\right. \\
& \varrho_{k+1} \mathrm{\varrho}_{k}^{-1}, \varrho_{k+3} \mathrm{\varrho}_{k+2}^{-1}, \cdots, \varrho_{2 k+2} \mathrm{\varrho}_{2 k+1}^{-1}, \varrho_{3 k+2} \mathrm{\varrho}_{3 k+1}^{-1}, \cdots, \\
& \left.\varrho_{3 k+3} \varrho_{3 k+2}^{-1}, \varrho_{k+2} \varrho_{3 k+3}^{-1} \frac{\alpha_{13}}{\alpha_{14}}, \varrho_{2 k+3} \varrho_{k+1}^{-1} \frac{\alpha_{16}}{\alpha_{17}}\right\}<1 .
\end{aligned}
$$

From (39), we get the required result.

In a similar way, dynamical characteristics of discrete systems (12)-(14) around $P_{0}$ can be investigated.

\subsection{Local Dynamical Characteristics of Discrete} Systems (12)-(14)

Theorem 2. For local dynamical characteristics about $P_{0}$ of discrete systems (12)-(14), the following statements hold:

(i) $P_{0}$ of (12) is a sink if

$$
\begin{aligned}
& \frac{\alpha_{19}}{\alpha_{20}}<1, \\
& \frac{\alpha_{22}}{\alpha_{23}}<1, \\
& \frac{\alpha_{25}}{\alpha_{26}}<1
\end{aligned}
$$


(ii) $P_{0}$ of (13) is a sink if

$$
\begin{aligned}
& \frac{\alpha_{28}}{\alpha_{29}}<1, \\
& \frac{\alpha_{31}}{\alpha_{32}}<1, \\
& \frac{\alpha_{34}}{\alpha_{35}}<1
\end{aligned}
$$

(iii) $P_{0}$ of (14) is a sink if

$$
\begin{aligned}
\frac{\alpha_{37}}{\alpha_{38}}<1, & \alpha_{40} \\
\alpha_{41} & <1, \\
\frac{\alpha_{43}}{\alpha_{44}} & <1
\end{aligned}
$$

Proof. Its proof is the same as the proof of Theorem 1.

\section{Boundedness of Discrete Systems (11)-(14)}

The boundedness of discrete systems (11)-(14) is explored in this section.

\subsection{Boundedness of Discrete System (11)}

Theorem 3. If $X_{n}=\left\{\left(x_{n}, y_{n}, z_{n}\right)\right\}$ is the positive solution of discrete system (11), then for $\mu \geq 0$, the following holds:

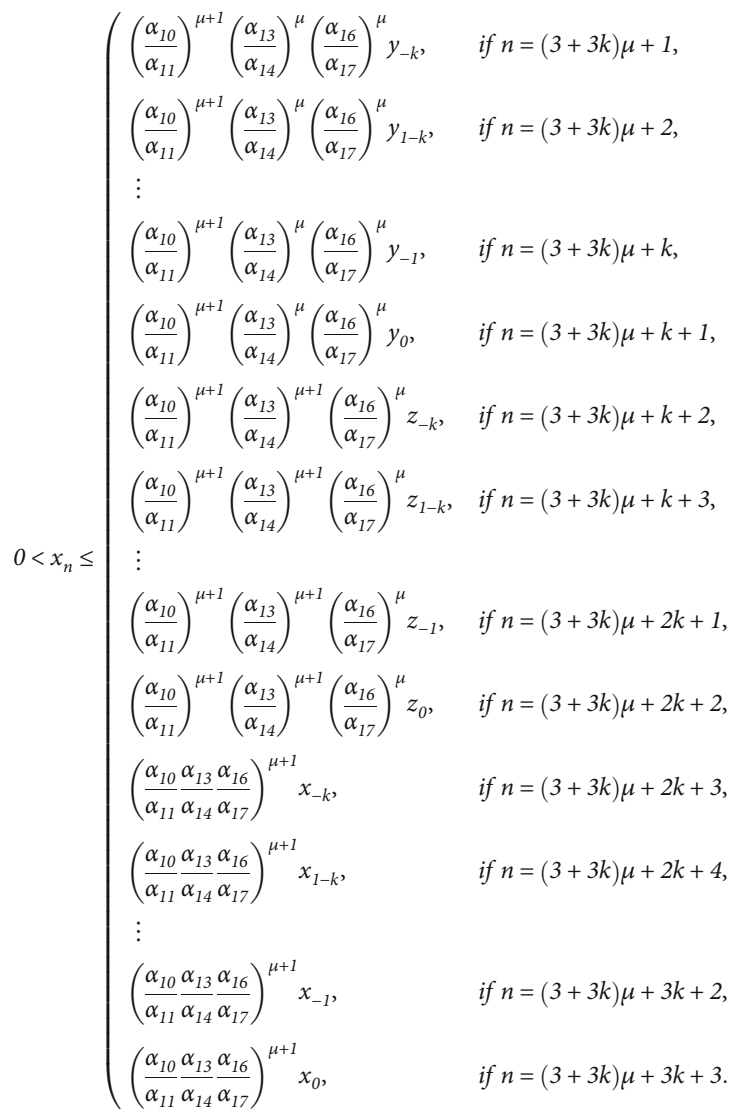

$$
\begin{aligned}
& \left(\left(\frac{\alpha_{10}}{\alpha_{11}}\right)^{\mu}\left(\frac{\alpha_{13}}{\alpha_{14}}\right)^{\mu+1}\left(\frac{\alpha_{16}}{\alpha_{17}}\right)^{\mu} z_{-k}, \quad \text { if } n=(3+3 k) \mu+1,\right. \\
& \left(\frac{\alpha_{10}}{\alpha_{11}}\right)^{\mu}\left(\frac{\alpha_{13}}{\alpha_{14}}\right)^{\mu+1}\left(\frac{\alpha_{16}}{\alpha_{17}}\right)^{\mu} z_{1-k}, \quad \text { if } n=(3+3 k) \mu+2, \\
& \vdots \\
& \left(\frac{\alpha_{10}}{\alpha_{11}}\right)^{\mu}\left(\frac{\alpha_{13}}{\alpha_{14}}\right)^{\mu+1}\left(\frac{\alpha_{16}}{\alpha_{17}}\right)^{\mu} z_{-1} \quad \text { if } n=(3+3 k) \mu+k \text {, } \\
& \left(\frac{\alpha_{10}}{\alpha_{11}}\right)^{\mu}\left(\frac{\alpha_{13}}{\alpha_{14}}\right)^{\mu+1}\left(\frac{\alpha_{16}}{\alpha_{17}}\right)^{\mu} z_{0}, \quad \text { if } n=(3+3 k) \mu+k+1 \text {, } \\
& \left(\frac{\alpha_{10}}{\alpha_{11}}\right)^{\mu}\left(\frac{\alpha_{13}}{\alpha_{14}}\right)^{\mu+1}\left(\frac{\alpha_{16}}{\alpha_{17}}\right)^{\mu+1} x_{-k}, \quad \text { if } n=(3+3 k) \mu+k+2 \text {, } \\
& \left(\frac{\alpha_{10}}{\alpha_{11}}\right)\left(\frac{\alpha_{13}}{\alpha_{14}}\right)^{\mu+1}\left(\frac{\alpha_{16}}{\alpha_{17}}\right)^{\mu+1} x_{1-k}, \quad \text { if } n=(3+3 k) \mu+k+3, \\
& 0<y_{n} \leq \\
& \left(\frac{\alpha_{10}}{\alpha_{11}}\right)^{\mu}\left(\frac{\alpha_{13}}{\alpha_{14}}\right)^{\mu+1}\left(\frac{\alpha_{16}}{\alpha_{17}}\right)^{\mu} x_{-1}, \quad \text { if } n=(3+3 k) \mu+2 k+1, \\
& \left(\frac{\alpha_{10}}{\alpha_{11}}\right)^{\mu}\left(\frac{\alpha_{13}}{\alpha_{14}}\right)^{\mu+1}\left(\frac{\alpha_{16}}{\alpha_{17}}\right)^{\mu} x_{0}, \quad \text { if } n=(3+3 k) \mu+2 k+2 \text {, } \\
& \left(\frac{\alpha_{10}}{\alpha_{11}} \frac{\alpha_{13}}{\alpha_{14}} \frac{\alpha_{16}}{\alpha_{17}}\right)^{\mu+1} y_{-k}, \quad \text { if } n=(3+3 k) \mu+2 k+3 \text {, } \\
& \left(\frac{\alpha_{10}}{\alpha_{11}} \frac{\alpha_{13}}{\alpha_{14}} \frac{\alpha_{16}}{\alpha_{17}}\right)^{\mu+1} y_{1-k}, \quad \text { if } n=(3+3 k) \mu+2 k+4 \text {, } \\
& \begin{array}{ll}
\left(\frac{\alpha_{10}}{\alpha_{11}} \frac{\alpha_{13}}{\alpha_{14}} \frac{\alpha_{16}}{\alpha_{17}}\right)^{\mu+1} y_{-1}, & \text { if } n=(3+3 k) \mu+3 k+2, \\
\left(\frac{\alpha_{10}}{\alpha_{11}} \frac{\alpha_{13}}{\alpha_{14}} \frac{\alpha_{16}}{\alpha_{17}}\right)^{\mu+1} y_{0}, & \text { if } n=(3+3 k) \mu+3 k+3 .
\end{array}
\end{aligned}
$$

Finally,

$$
\begin{aligned}
& \begin{cases}\left(\frac{\alpha_{10}}{\alpha_{11}}\right)^{\mu}\left(\frac{\alpha_{13}}{\alpha_{14}}\right)^{\mu}\left(\frac{\alpha_{16}}{\alpha_{17}}\right)^{\mu+1} x_{-k}, & \text { if } n=(3+3 k) \mu+1, \\
\left(\frac{\alpha_{10}}{\alpha_{11}}\right)^{\mu}\left(\frac{\alpha_{13}}{\alpha_{14}}\right)^{\mu}\left(\frac{\alpha_{16}}{\alpha_{17}}\right)^{\mu+1} x_{1-k}, & \text { if } n=(3+3 k) \mu+2,\end{cases} \\
& \left(\frac{\alpha_{10}}{\alpha_{11}}\right)^{\mu}\left(\frac{\alpha_{13}}{\alpha_{14}}\right)^{\mu}\left(\frac{\alpha_{16}}{\alpha_{17}}\right)^{\mu+1} x_{-1}, \quad \text { if } n=(3+3 k) \mu+k, \\
& \left(\frac{\alpha_{10}}{\alpha_{11}}\right)^{\mu}\left(\frac{\alpha_{13}}{\alpha_{14}}\right)^{\mu}\left(\frac{\alpha_{16}}{\alpha_{17}}\right)^{\mu+1} x_{0}, \quad \text { if } n=(3+3 k) \mu+k+1 \text {, } \\
& \left(\frac{\alpha_{10}}{\alpha_{11}}\right)^{\mu+1}\left(\frac{\alpha_{13}}{\alpha_{14}}\right)^{\mu}\left(\frac{\alpha_{16}}{\alpha_{17}}\right)^{\mu+1} y_{-k}, \quad \text { if } n=(3+3 k) \mu+k+2 \text {, } \\
& \left(\frac{\alpha_{10}}{\alpha_{11}}\right)^{\mu+1}\left(\frac{\alpha_{13}}{\alpha_{14}}\right)^{\mu}\left(\frac{\alpha_{16}}{\alpha_{17}}\right)^{\mu+1} y_{1-k}, \quad \text { if } n=(3+3 k) \mu+k+3, \\
& 0<z_{n} \leq \\
& \left(\frac{\alpha_{10}}{\alpha_{11}}\right)^{\mu+1}\left(\frac{\alpha_{13}}{\alpha_{14}}\right)^{\mu}\left(\frac{\alpha_{16}}{\alpha_{17}}\right)^{\mu+1} y_{-1}, \quad \text { if } n=(3+3 k) \mu+2 k+1, \\
& \left(\frac{\alpha_{10}}{\alpha_{11}}\right)^{\mu+1}\left(\frac{\alpha_{13}}{\alpha_{14}}\right)^{\mu}\left(\frac{\alpha_{16}}{\alpha_{17}}\right)^{\mu+1} y_{0}, \quad \text { if } n=(3+3 k) \mu+2 k+2, \\
& \left(\frac{\alpha_{10}}{\alpha_{11}} \frac{\alpha_{13}}{\alpha_{14}} \frac{\alpha_{16}}{\alpha_{17}}\right)^{\mu+1} z_{-k}, \quad \text { if } n=(3+3 k) \mu+2 k+3 \text {, } \\
& \left(\frac{\alpha_{10}}{\alpha_{11}} \frac{\alpha_{13}}{\alpha_{14}} \frac{\alpha_{16}}{\alpha_{17}}\right)^{\mu+1} z_{1-k}, \quad \text { if } n=(3+3 k) \mu+2 k+4, \\
& \vdots \\
& \left(\frac{\alpha_{10}}{\alpha_{11}} \frac{\alpha_{13}}{\alpha_{14}} \frac{\alpha_{16}}{\alpha_{17}}\right)^{\mu+1} z_{-1}, \quad \text { if } n=(3+3 k) \mu+3 k+2, \\
& \left(\frac{\alpha_{10}}{\alpha_{11}} \frac{\alpha_{13}}{\alpha_{14}} \frac{\alpha_{16}}{\alpha_{17}}\right)^{\mu+1} z_{0}, \quad \text { if } n=(3+3 k) \mu+3 k+3 \text {. }
\end{aligned}
$$


Proof. Noticeably, (43), (44), and (45) hold if $\mu=0$. If (43), (44), and (45) are true for $\mu=\sigma$, i.e.,

$$
\left\{\begin{array}{lll}
\left(\frac{\alpha_{10}}{\alpha_{11}}\right)^{\sigma+1}\left(\frac{\alpha_{13}}{\alpha_{14}}\right)^{\sigma}\left(\frac{\alpha_{16}}{\alpha_{17}}\right)^{\sigma} y_{-k}, & \text { if } n=(3+3 k) \sigma+1, \\
\left(\frac{\alpha_{10}}{\alpha_{11}}\right)^{\sigma+1}\left(\frac{\alpha_{13}}{\alpha_{14}}\right)^{\sigma}\left(\frac{\alpha_{16}}{\alpha_{17}}\right)^{\sigma} y_{1-k}, & \text { if } n=(3+3 k) \sigma+2, \\
\vdots & \left(\frac{\alpha_{10}}{\alpha_{11}}\right)^{\sigma+1}\left(\frac{\alpha_{13}}{\alpha_{14}}\right)^{\sigma}\left(\frac{\alpha_{16}}{\alpha_{17}}\right)^{\sigma} y_{-1}, & \text { if } n=(3+3 k) \sigma+k, \\
\left(\frac{\alpha_{10}}{\alpha_{11}}\right)^{\sigma+1}\left(\frac{\alpha_{13}}{\alpha_{14}}\right)^{\sigma}\left(\frac{\alpha_{16}}{\alpha_{17}}\right)^{\sigma} y_{0}, & \text { if } n=(3+3 k) \sigma+k+1, \\
\left(\frac{\alpha_{10}}{\alpha_{11}}\right)^{\sigma+1}\left(\frac{\alpha_{13}}{\alpha_{14}}\right)^{\sigma+1}\left(\frac{\alpha_{16}}{\alpha_{17}}\right)^{\sigma} z_{-k}, & \text { if } n=(3+3 k) \sigma+k+2, \\
\left(\frac{\alpha_{10}}{\alpha_{11}}\right)^{\sigma+1}\left(\frac{\alpha_{13}}{\alpha_{14}}\right)^{\sigma+1}\left(\frac{\alpha_{16}}{\alpha_{17}}\right)^{\sigma} z_{1-k}, & \text { if } n=(3+3 k) \sigma+k+3, \\
\left(\frac{\alpha_{10}}{\alpha_{11}}\right)^{\sigma+1}\left(\frac{\alpha_{13}}{\alpha_{14}}\right)^{\sigma+1}\left(\frac{\alpha_{16}}{\alpha_{17}}\right)^{\sigma} z_{-1}, & \text { if } n=(3+3 k) \sigma+2 k+1, \\
\left(\frac{\alpha_{10}}{\alpha_{11}}\right)^{\sigma+1}\left(\frac{\alpha_{13}}{\alpha_{14}}\right)^{\sigma+1}\left(\frac{\alpha_{16}}{\alpha_{17}}\right)^{\sigma} z_{0}, & \text { if } n=(3+3 k) \sigma+2 k+2, \\
\left(\frac{\alpha_{10}}{\alpha_{11}} \frac{\alpha_{13}}{\alpha_{14}} \frac{\alpha_{16}}{\alpha_{17}}\right)^{\sigma+1} x_{-k}, & \text { if } n=(3+3 k) \sigma+2 k+3, \\
\left(\frac{\alpha_{10}}{\alpha_{11}} \frac{\alpha_{13}}{\alpha_{14}} \frac{\alpha_{16}}{\alpha_{17}}\right)^{\sigma+1} x_{1-k}, & \text { if } n=(3+3 k) \sigma+2 k+4, \\
\vdots & \\
\left(\frac{\alpha_{10}}{\alpha_{11}} \frac{\alpha_{13}}{\alpha_{14}} \frac{\alpha_{16}}{\alpha_{11}} \frac{\alpha_{13}}{\alpha_{14}} \frac{\alpha_{16}}{\alpha_{17}}\right)^{\sigma+1} x_{-1}, & \text { if } n=(3+3 k) \sigma+3 k+2, \\
x_{0}, &
\end{array}\right.
$$$$
\left\{\begin{array}{lll}
\left(\frac{\alpha_{10}}{\alpha_{11}}\right)^{\sigma}\left(\frac{\alpha_{13}}{\alpha_{14}}\right)^{\sigma+1}\left(\frac{\alpha_{16}}{\alpha_{17}}\right)^{\sigma} z_{-k}, & \text { if } n=(3+3 k) \sigma+1, \\
\left(\frac{\alpha_{10}}{\alpha_{11}}\right)^{\sigma}\left(\frac{\alpha_{13}}{\alpha_{14}}\right)^{\sigma+1}\left(\frac{\alpha_{16}}{\alpha_{17}}\right)^{\sigma} z_{1-k}, & \text { if } n=(3+3 k) \sigma+2, \\
\vdots & \left(\frac{\alpha_{10}}{\alpha_{11}}\right)^{\sigma}\left(\frac{\alpha_{13}}{\alpha_{14}}\right)^{\sigma+1}\left(\frac{\alpha_{16}}{\alpha_{17}}\right)^{\sigma} z_{-1}, & \text { if } n=(3+3 k) \sigma+k, \\
\left(\frac{\alpha_{10}}{\alpha_{11}}\right)^{\sigma}\left(\frac{\alpha_{13}}{\alpha_{14}}\right)^{\sigma+1}\left(\frac{\alpha_{16}}{\alpha_{17}}\right)^{\sigma} z_{0}, & \text { if } n=(3+3 k) \sigma+k+1, \\
\left(\frac{\alpha_{10}}{\alpha_{11}}\right)^{\sigma}\left(\frac{\alpha_{13}}{\alpha_{14}}\right)^{\sigma+1}\left(\frac{\alpha_{16}}{\alpha_{17}}\right)^{\sigma+1} x_{-k}, & \text { if } n=(3+3 k) \sigma+k+2, \\
\left.\left(\frac{\alpha_{10}}{\alpha_{11}}\right)^{\frac{\alpha_{13}}{\alpha_{14}}}\right)^{\sigma+1}\left(\frac{\alpha_{16}}{\alpha_{17}}\right)^{\sigma+1} x_{1-k}, & \text { if } n=(3+3 k) \sigma+k+3, \\
\left(\frac{\alpha_{10}}{\alpha_{11}}\right)^{\sigma}\left(\frac{\alpha_{13}}{\alpha_{14}}\right)^{\sigma+1}\left(\frac{\alpha_{16}}{\alpha_{17}}\right)^{\sigma} x_{-1}, & \text { if } n=(3+3 k) \sigma+2 k+1, \\
\left(\frac{\alpha_{10}}{\alpha_{11}}\right)^{\sigma}\left(\frac{\alpha_{13}}{\alpha_{14}}\right)^{\sigma+1}\left(\frac{\alpha_{16}}{\alpha_{17}}\right)^{\sigma} x_{0}, & \text { if } n=(3+3 k) \sigma+2 k+2, \\
\left(\frac{\alpha_{10}}{\alpha_{11}} \frac{\alpha_{13}}{\alpha_{14}} \frac{\alpha_{16}}{\alpha_{17}}\right)^{\sigma+1} y_{-k}, & \text { if } n=(3+3 k) \sigma+2 k+3, \\
\left(\frac{\alpha_{10}}{\alpha_{11}} \frac{\alpha_{13}}{\alpha_{14}} \frac{\alpha_{16}}{\alpha_{17}}\right)^{\sigma+1} y_{1-k}, & \text { if } n=(3+3 k) \sigma+2 k+4, \\
\vdots \\
\left.\left(\frac{\alpha_{10}}{\alpha_{11}} \frac{\alpha_{13}}{\alpha_{14}} \frac{\alpha_{16}}{\alpha_{17}}\right)^{\sigma+1} y_{-1}, \frac{\alpha_{16}}{\alpha_{14}} \frac{\alpha_{17}}{\alpha_{17}}\right)^{\sigma+1} y_{0}, & \text { if } n=(3+3 k) \sigma+3 k+2,
\end{array}\right.
$$

Finally,

$$
\begin{aligned}
& \begin{cases}\left(\frac{\alpha_{10}}{\alpha_{11}}\right)^{\sigma}\left(\frac{\alpha_{13}}{\alpha_{14}}\right)^{\sigma}\left(\frac{\alpha_{16}}{\alpha_{17}}\right)^{\sigma+1} x_{-k}, & \text { if } n=(3+3 k) \sigma+1, \\
\left(\frac{\alpha_{10}}{\alpha_{11}}\right)^{\sigma}\left(\frac{\alpha_{13}}{\alpha_{14}}\right)^{\sigma}\left(\frac{\alpha_{16}}{\alpha_{17}}\right)^{\sigma+1} x_{1-k}, & \text { if } n=(3+3 k) \sigma+2,\end{cases} \\
& \vdots \\
& \left(\frac{\alpha_{10}}{\alpha_{11}}\right)^{\sigma}\left(\frac{\alpha_{13}}{\alpha_{14}}\right)^{\sigma}\left(\frac{\alpha_{16}}{\alpha_{17}}\right)^{\sigma+1} x_{-1}, \quad \text { if } n=(3+3 k) \sigma+k \text {, } \\
& \left(\frac{\alpha_{10}}{\alpha_{11}}\right)^{\sigma}\left(\frac{\alpha_{13}}{\alpha_{14}}\right)^{\sigma}\left(\frac{\alpha_{16}}{\alpha_{17}}\right)^{\sigma+1} x_{0}, \quad \text { if } n=(3+3 k) \sigma+k+1 \text {, } \\
& \left(\frac{\alpha_{10}}{\alpha_{11}}\right)^{\sigma+1}\left(\frac{\alpha_{13}}{\alpha_{14}}\right)^{\sigma}\left(\frac{\alpha_{16}}{\alpha_{17}}\right)^{\sigma+1} y_{-k}, \quad \text { if } n=(3+3 k) \sigma+k+2 \text {, } \\
& \left(\frac{\alpha_{10}}{\alpha_{11}}\right)^{\sigma+1}\left(\frac{\alpha_{13}}{\alpha_{14}}\right)^{\sigma}\left(\frac{\alpha_{16}}{\alpha_{17}}\right)^{\sigma+1} y_{1-k}, \quad \text { if } n=(3+3 k) \sigma+k+3, \\
& 0<z_{n} \leq \quad \text { : } \\
& \left(\frac{\alpha_{10}}{\alpha_{11}}\right)^{\sigma+1}\left(\frac{\alpha_{13}}{\alpha_{14}}\right)^{\sigma}\left(\frac{\alpha_{16}}{\alpha_{17}}\right)^{\sigma+1} y_{-1}, \quad \text { if } n=(3+3 k) \sigma+2 k+1, \\
& \left(\frac{\alpha_{10}}{\alpha_{11}}\right)^{\sigma+1}\left(\frac{\alpha_{13}}{\alpha_{14}}\right)^{\sigma}\left(\frac{\alpha_{16}}{\alpha_{17}}\right)^{\sigma+1} y_{0}, \quad \text { if } n=(3+3 k) \sigma+2 k+2 \text {, } \\
& \left(\frac{\alpha_{10}}{\alpha_{11}} \frac{\alpha_{13}}{\alpha_{14}} \frac{\alpha_{16}}{\alpha_{17}}\right)^{\sigma+1} z_{-k}, \quad \text { if } n=(3+3 k) \sigma+2 k+3 \text {, } \\
& \left(\frac{\alpha_{10}}{\alpha_{11}} \frac{\alpha_{13}}{\alpha_{14}} \frac{\alpha_{16}}{\alpha_{17}}\right)^{\sigma+1} z_{1-k}, \quad \text { if } n=(3+3 k) \sigma+2 k+4 \text {, } \\
& \vdots \\
& \left(\frac{\alpha_{10}}{\alpha_{11}} \frac{\alpha_{13}}{\alpha_{14}} \frac{\alpha_{16}}{\alpha_{17}}\right)^{\sigma+1} z_{-1}, \quad \text { if } n=(3+3 k) \sigma+3 k+2 \text {, } \\
& \left(\frac{\alpha_{10}}{\alpha_{11}} \frac{\alpha_{13}}{\alpha_{14}} \frac{\alpha_{16}}{\alpha_{17}}\right)^{\sigma+1} z_{0}, \quad \text { if } n=(3+3 k) \sigma+3 k+3 \text {. }
\end{aligned}
$$

Than for $\mu=\sigma+1$, we have

$$
\begin{aligned}
x_{(3 k+3)(\sigma+1)+1} & =\frac{\alpha_{10} y_{(3 k+3) \sigma+2 k+3}}{\alpha_{11}+\alpha_{12} \prod_{i=0}^{k} z_{(3 k+3) \sigma+3 k+3-i}}, \leq \frac{\alpha_{10}}{\alpha_{11}} y_{(3 k+3) \sigma+2 k+3} \\
& \leq\left(\frac{\alpha_{10}}{\alpha_{11}}\right)^{\sigma+2}\left(\frac{\alpha_{13}}{\alpha_{14}}\right)^{\sigma+1}\left(\frac{\alpha_{16}}{\alpha_{17}}\right)^{\sigma+1} y_{-k},
\end{aligned}
$$

$$
\begin{aligned}
& x_{(3 k+3)(\sigma+1)+2}=\frac{\alpha_{10} y_{(3 k+3) \sigma+2 k+4}}{\alpha_{11}+\alpha_{12} \prod_{i=0}^{k} z_{(3 k+3) \sigma+3 k+4-i}}, \leq \frac{\alpha_{10}}{\alpha_{11}} y_{(3 k+3) \sigma+2 k+4} \\
& \leq\left(\frac{\alpha_{10}}{\alpha_{11}}\right)^{\sigma+2}\left(\frac{\alpha_{13}}{\alpha_{14}}\right)^{\sigma+1}\left(\frac{\alpha_{16}}{\alpha_{17}}\right)^{\sigma+1} y_{-k+1}, \\
& \vdots \\
& x_{(3 k+3)(\sigma+1)+k}=\frac{\alpha_{10} y_{(3 k+3) \sigma+3 k+2}}{\alpha_{11}+\alpha_{12} \prod_{i=0}^{k} z_{(3 k+3) \sigma+4 k+2-i}}, \leq \frac{\alpha_{10}}{\alpha_{11}} y_{(3 k+3) \sigma+3 k+2} \\
& \leq\left(\frac{\alpha_{10}}{\alpha_{11}}\right)^{\sigma+2}\left(\frac{\alpha_{13}}{\alpha_{14}}\right)^{\sigma+1}\left(\frac{\alpha_{16}}{\alpha_{17}}\right)^{\sigma+1} y_{-1},
\end{aligned}
$$




$$
\begin{aligned}
x_{(3 k+3)(\sigma+1)+k+1} & =\frac{\alpha_{10} y_{(3 k+3) \sigma+3 k+3}}{\alpha_{11}+\alpha_{12} \prod_{i=0}^{k} z_{(3 k+3) \sigma+4 k+3-i}}, \leq \frac{\alpha_{10}}{\alpha_{11}} y_{(3 k+3) \sigma+3 k+3} \\
& \leq\left(\frac{\alpha_{10}}{\alpha_{11}}\right)^{\sigma+2}\left(\frac{\alpha_{13}}{\alpha_{14}}\right)^{\sigma+1}\left(\frac{\alpha_{16}}{\alpha_{17}}\right)^{\sigma+1} y_{0}, \\
y_{(3 k+3)(\sigma+1)+1} & =\frac{\alpha_{13} z_{(3 k+3) \sigma+2 k+3}}{\alpha_{14}+\alpha_{15} \prod_{i=0}^{k} x_{(3 k+3) \sigma+3 k+3-i}}, \leq \frac{\alpha_{13}}{\alpha_{14}} z_{(3 k+3) \sigma+2 k+3} \\
& \leq\left(\frac{\alpha_{10}}{\alpha_{11}}\right)^{\sigma+1}\left(\frac{\alpha_{13}}{\alpha_{14}}\right)^{\sigma+2}\left(\frac{\alpha_{16}}{\alpha_{17}}\right)^{\sigma+1} z_{-k}, \\
y_{(3 k+3)(\sigma+1)+2} & =\frac{\alpha_{13} z_{(3 k+3) \sigma+2 k+4}}{\alpha_{14}+\alpha_{15} \prod_{i=0}^{k} x_{(3 k+3) \sigma+3 k+4-i}}, \leq \frac{\alpha_{13}}{\alpha_{14}} z_{(3 k+3) \sigma+2 k+4} \\
& \leq\left(\frac{\alpha_{10}}{\alpha_{11}}\right)^{\sigma+1}\left(\frac{\alpha_{13}}{\alpha_{14}}\right)^{\sigma+2}\left(\frac{\alpha_{16}}{\alpha_{17}}\right)^{\sigma+1} z_{-k+1}, \\
& \leq\left(\frac{\alpha_{10}}{\alpha_{11}}\right)^{\sigma+1}\left(\frac{\alpha_{13}}{\alpha_{14}}\right)^{\sigma+2}\left(\frac{\alpha_{16}}{\alpha_{17}}\right)^{\sigma+1} z_{-1}, \\
y_{(3 k+3)(\sigma+1)+k} & =\frac{\alpha_{13} z_{(3 k+3) \sigma+3 k+2}}{\alpha_{14}+\alpha_{15} \prod_{i=0}^{k} x_{(3 k+3) \sigma+4 k+2-i}} z_{(3 k+3) \sigma+3 k+2}
\end{aligned}
$$

$y_{(3 k+3)(\sigma+1)+k+1}=\frac{\alpha_{13} z_{(3 k+3) \sigma+3 k+3}}{\alpha_{14}+\alpha_{15} \prod_{i=0}^{k} x_{(3 k+3) \sigma+4 k+3-i}}, \leq \frac{\alpha_{13}}{\alpha_{14}} z_{(3 k+3) \sigma+3 k+3}$

$$
\leq\left(\frac{\alpha_{10}}{\alpha_{11}}\right)^{\sigma+1}\left(\frac{\alpha_{13}}{\alpha_{14}}\right)^{\sigma+2}\left(\frac{\alpha_{16}}{\alpha_{17}}\right)^{\sigma+1} z_{0}
$$

$z_{(3 k+3)(\sigma+1)+1}=\frac{\alpha_{16} x_{(3 k+3) \sigma+2 k+3}}{\alpha_{17}+\alpha_{18} \prod_{i=0}^{k} y_{(3 k+3) \sigma+3 k+3-i}}, \leq \frac{\alpha_{16}}{\alpha_{17}} x_{(3 k+3) \sigma+2 k+3}$ $\leq\left(\frac{\alpha_{10}}{\alpha_{11}}\right)^{\sigma+1}\left(\frac{\alpha_{13}}{\alpha_{14}}\right)^{\sigma+1}\left(\frac{\alpha_{16}}{\alpha_{17}}\right)^{\sigma+2} x_{-k}$

$z_{(3 k+3)(\sigma+1)+2}=\frac{\alpha_{16} x_{(3 k+3) \sigma+2 k+4}}{\alpha_{17}+\alpha_{18} \prod_{i=0}^{k} y_{(3 k+3) \sigma+3 k+4-i}}, \leq \frac{\alpha_{16}}{\alpha_{17}} x_{(3 k+3) \sigma+2 k+4}$ $\leq\left(\frac{\alpha_{10}}{\alpha_{11}}\right)^{\sigma+1}\left(\frac{\alpha_{13}}{\alpha_{14}}\right)^{\sigma+1}\left(\frac{\alpha_{16}}{\alpha_{17}}\right)^{\sigma+2} x_{-k+1}$,

$z_{(3 k+3)(\sigma+1)+k}=\frac{\alpha_{16} x_{(3 k+3) \sigma+3 k+2}}{\alpha_{17}+\alpha_{18} \prod_{i=0}^{k} y_{(3 k+3) \sigma+4 k+2-i}}, \leq \frac{\alpha_{16}}{\alpha_{17}} x_{(3 k+3) \sigma+3 k+2}$ $\leq\left(\frac{\alpha_{10}}{\alpha_{11}}\right)^{\sigma+1}\left(\frac{\alpha_{13}}{\alpha_{14}}\right)^{\sigma+1}\left(\frac{\alpha_{16}}{\alpha_{17}}\right)^{\sigma+2} x_{-1}$,

$z_{(3 k+3)(\sigma+1)+k+1}=\frac{\alpha_{16} x_{(3 k+3) \sigma+3 k+3}}{\alpha_{17}+\alpha_{18} \prod_{i=0}^{k} y_{(3 k+3) \sigma+4 k+3-i}}, \leq \frac{\alpha_{16}}{\alpha_{17}} x_{(3 k+3) \sigma+3 k+3}$ $\leq\left(\frac{\alpha_{10}}{\alpha_{11}}\right)^{\sigma+1}\left(\frac{\alpha_{13}}{\alpha_{14}}\right)^{\sigma+1}\left(\frac{\alpha_{16}}{\alpha_{17}}\right)^{\sigma+2} x_{0}$.

\section{And}

$$
\begin{aligned}
x_{(3 k+3)(\sigma+1)+k+2} & =\frac{\alpha_{10} y_{(3 k+3)(\sigma+1)+1}}{\alpha_{11}+\alpha_{12} \prod_{i=0}^{k} z_{(3 k+3) \sigma+4 k+4-i}}, \leq \frac{\alpha_{10}}{\alpha_{11}} y_{(3 k+3)(\sigma+1)+1} \\
& \leq\left(\frac{\alpha_{10}}{\alpha_{11}}\right)^{\sigma+2}\left(\frac{\alpha_{13}}{\alpha_{14}}\right)^{\sigma+2}\left(\frac{\alpha_{16}}{\alpha_{17}}\right)^{\sigma+1} z_{-k}, \\
x_{(3 k+3)(\sigma+1)+k+3} & =\frac{\alpha_{10} y_{(3 k+3)(\sigma+1)+2}}{\alpha_{11}+\alpha_{12} \prod_{i=0}^{k} z_{(3 k+3) \sigma+4 k+5-i}}, \leq \frac{\alpha_{10}}{\alpha_{11}} y_{(3 k+3)(\sigma+1)+2} \\
& \leq\left(\frac{\alpha_{10}}{\alpha_{11}}\right)^{\sigma+2}\left(\frac{\alpha_{13}}{\alpha_{14}}\right)^{\sigma+2}\left(\frac{\alpha_{16}}{\alpha_{17}}\right)^{\sigma+1} z_{-k+1}, \\
x_{(3 k+3)(\sigma+1)+2 k+1} & =\frac{\alpha_{10} y_{(3 k+3)(\sigma+1)+k}}{\alpha_{11}+\alpha_{12} \prod_{i=0}^{k} z_{(3 k+3) \sigma+5 k+3-i}}, \leq \frac{\alpha_{10}}{\alpha_{11}} y_{(3 k+3)(\sigma+1)+k} \\
& \leq\left(\frac{\alpha_{10}}{\alpha_{11}}\right)^{\sigma+2}\left(\frac{\alpha_{13}}{\alpha_{14}}\right)^{\sigma+2}\left(\frac{\alpha_{16}}{\alpha_{17}}\right)^{\sigma+1} z_{-1},
\end{aligned}
$$

$x_{(3 k+3)(\sigma+1)+2 k+2}=\frac{\alpha_{10} y_{(3 k+3)(\sigma+1)+k+1}}{\alpha_{11}+\alpha_{12} \prod_{i=0}^{k} z_{(3 k+3) \sigma+5 k+4-i}}, \leq \frac{\alpha_{10}}{\alpha_{11}} y_{(3 k+3)(\sigma+1)+2}$ $\leq\left(\frac{\alpha_{10}}{\alpha_{11}}\right)^{\sigma+2}\left(\frac{\alpha_{13}}{\alpha_{14}}\right)^{\sigma+2}\left(\frac{\alpha_{16}}{\alpha_{17}}\right)^{\sigma+1} z_{0}$

$y_{(3 k+3)(\sigma+1)+k+2}=\frac{\alpha_{13} z_{(3 k+3)(\sigma+1)+1}}{\alpha_{14}+\alpha_{15} \prod_{i=0}^{k} x_{(3 k+3) \sigma+4 k+4-i}}, \leq \frac{\alpha_{13}}{\alpha_{14}} z_{(3 k+3)(\sigma+1)+1}$ $\leq\left(\frac{\alpha_{10}}{\alpha_{11}}\right)^{\sigma+1}\left(\frac{\alpha_{13}}{\alpha_{14}}\right)^{\sigma+2}\left(\frac{\alpha_{16}}{\alpha_{17}}\right)^{\sigma+2} x_{-k}$

$y_{(3 k+3)(\sigma+1)+k+3}=\frac{\alpha_{13} z_{(3 k+3)(\sigma+1)+2}}{\alpha_{14}+\alpha_{15} \prod_{i=0}^{k} x_{(3 k+3) \sigma+4 k+5-i}}, \leq \frac{\alpha_{13}}{\alpha_{14}} z_{(3 k+3)(\sigma+1)+2}$ $\leq\left(\frac{\alpha_{10}}{\alpha_{11}}\right)^{\sigma+1}\left(\frac{\alpha_{13}}{\alpha_{14}}\right)^{\sigma+2}\left(\frac{\alpha_{16}}{\alpha_{17}}\right)^{\sigma+2} x_{-k+1}$,

$y_{(3 k+3)(\sigma+1)+2 k+1}=\frac{\alpha_{13} z_{(3 k+3)(\sigma+1)+k}}{\alpha_{14}+\alpha_{15} \prod_{i=0}^{k} x_{(3 k+3) \sigma+5 k+3-i}}, \leq \frac{\alpha_{13}}{\alpha_{14}} z_{(3 k+3)(\sigma+1)+k}$ $\leq\left(\frac{\alpha_{10}}{\alpha_{11}}\right)^{\sigma+1}\left(\frac{\alpha_{13}}{\alpha_{14}}\right)^{\sigma+2}\left(\frac{\alpha_{16}}{\alpha_{17}}\right)^{\sigma+2} x_{-1}$,

$y_{(3 k+3)(\sigma+1)+2 k+2}=\frac{\alpha_{13} z_{(3 k+3)(\sigma+1)+k+1}}{\alpha_{14}+\alpha_{15} \prod_{i=0}^{k} x_{(3 k+3) \sigma+5 k+4-i}}, \leq \frac{\alpha_{13}}{\alpha_{14}} z_{(3 k+3)(\sigma+1)+k+1}$ $\leq\left(\frac{\alpha_{10}}{\alpha_{11}}\right)^{\sigma+1}\left(\frac{\alpha_{13}}{\alpha_{14}}\right)^{\sigma+2}\left(\frac{\alpha_{16}}{\alpha_{17}}\right)^{\sigma+2} x_{0}$,

$z_{(3 k+3)(\sigma+1)+k+2}=\frac{\alpha_{16} x_{(3 k+3)(\sigma+1)+1}}{\alpha_{17}+\alpha_{18} \prod_{i=0}^{k} y_{(3 k+3) \sigma+4 k+4-i}}, \leq \frac{\alpha_{16}}{\alpha_{17}} x_{(3 k+3)(\sigma+1)+1}$ $\leq\left(\frac{\alpha_{10}}{\alpha_{11}}\right)^{\sigma+2}\left(\frac{\alpha_{13}}{\alpha_{14}}\right)^{\sigma+1}\left(\frac{\alpha_{16}}{\alpha_{17}}\right)^{\sigma+2} y_{-k}$, 


$$
\begin{aligned}
z_{(3 k+3)(\sigma+1)+k+3} & =\frac{\alpha_{16} x_{(3 k+3)(\sigma+1)+2}}{\alpha_{17}+\alpha_{18} \prod_{i=0}^{k} y_{(3 k+3) \sigma+4 k+5-i}}, \leq \frac{\alpha_{16}}{\alpha_{17}} x_{(3 k+3)(\sigma+1)+2} \\
& \leq\left(\frac{\alpha_{10}}{\alpha_{11}}\right)^{\sigma+2}\left(\frac{\alpha_{13}}{\alpha_{14}}\right)^{\sigma+1}\left(\frac{\alpha_{16}}{\alpha_{17}}\right)^{\sigma+2} y_{-k+1}, \\
\vdots & \\
z_{(3 k+3)(\sigma+1)+2 k+1} & =\frac{\alpha_{16} x_{(3 k+3)(\sigma+1)+k}}{\alpha_{17}+\alpha_{18} \prod_{i=0}^{k} y_{(3 k+3) \sigma+5 k+3-i}}, \leq \frac{\alpha_{16}}{\alpha_{17}} x_{(3 k+3)(\sigma+1)+k} \\
& \leq\left(\frac{\alpha_{10}}{\alpha_{11}}\right)^{\sigma+2}\left(\frac{\alpha_{13}}{\alpha_{14}}\right)^{\sigma+1}\left(\frac{\alpha_{16}}{\alpha_{17}}\right)^{\sigma+2} y_{-1}, \\
z_{(3 k+3)(\sigma+1)+2 k+2} & =\frac{\alpha_{16} x_{(3 k+3)(\sigma+1)+k+1}}{\alpha_{17}+\alpha_{18} \prod_{i=0}^{k} y_{(3 k+3) \sigma+5 k+4-i}}, \leq \frac{\alpha_{16}}{\alpha_{17}} x_{(3 k+3)(\sigma+1)+k+1} \\
& \leq\left(\frac{\alpha_{10}}{\alpha_{11}}\right)^{\sigma+2}\left(\frac{\alpha_{13}}{\alpha_{14}}\right)^{\sigma+1}\left(\frac{\alpha_{16}}{\alpha_{17}}\right)^{\sigma+2} y_{0} .
\end{aligned}
$$

Finally,

$$
\begin{aligned}
x_{(3 k+3)(\sigma+1)+2 k+3} & =\frac{\alpha_{10} y_{(3 k+3)(\sigma+1)+k+2}}{\alpha_{11}+\alpha_{12} \prod_{i=0}^{k} z_{(3 k+3) \sigma+5 k+5-i}}, \\
& \leq \frac{\alpha_{10}}{\alpha_{11}} y_{(3 k+3)(\sigma+1)+k+2} \leq\left(\frac{\alpha_{10}}{\alpha_{11}} \frac{\alpha_{13}}{\alpha_{14}} \frac{\alpha_{16}}{\alpha_{17}}\right)^{\sigma+2} x_{-k}, \\
x_{(3 k+3)(\sigma+1)+2 k+4} & =\frac{\alpha_{10} y_{(3 k+3)(\sigma+1)+k+3}}{\alpha_{11}+\alpha_{12} \prod_{i=0}^{k} z_{(3 k+3) \sigma+5 k+6-i}}, \\
& \leq \frac{\alpha_{10}}{\alpha_{11}} y_{(3 k+3)(\sigma+1)+k+3} \leq\left(\frac{\alpha_{10}}{\alpha_{11}} \frac{\alpha_{13}}{\alpha_{14}} \frac{\alpha_{16}}{\alpha_{17}}\right)^{\sigma+2} x_{-k+1}, \\
y_{(3 k+3)(\sigma+1)+2 k+3} & =\frac{\alpha_{13} z_{(3 k+3)(\sigma+1)+k+2}}{\alpha_{14}+\alpha_{15} \prod_{i=0}^{k} x_{(3 k+3) \sigma+5 k+5-i}}, \\
& \leq \frac{\alpha_{13}}{\alpha_{14}} z_{(3 k+3)(\sigma+1)+k+2} \leq\left(\frac{\alpha_{10}}{\alpha_{11}} \frac{\alpha_{13}}{\alpha_{14}} \frac{\alpha_{16}}{\alpha_{17}}\right)^{\sigma+2} y_{-k}, \\
& \leq \frac{\alpha_{10} y_{(3 k+3)(\sigma+1)+2 k+1}}{\alpha_{11}} y_{(3 k+3)(\sigma+1)+2 k+2} \leq\left(\frac{\alpha_{10}}{\alpha_{11}} \frac{\alpha_{13}}{\alpha_{14}} \frac{\alpha_{16}}{\alpha_{17}}\right)^{\sigma+2} y_{(3 k+3)(\sigma+1)+2 k+1} \leq\left(\frac{\alpha_{10}}{\alpha_{11}} \frac{\alpha_{13}}{\alpha_{14}} \frac{\alpha_{16}}{\alpha_{17}}\right)^{\sigma+2} x_{-1}, \\
x_{(3 k+3)(\sigma+1)+3 k+3} & =\frac{\alpha_{10} y_{(3 k+3)(\sigma+1)+2 k+2}}{\alpha_{11}+\alpha_{12} \prod_{i=0}^{k} z_{(3 k+3) \sigma+6 k+5-i}},
\end{aligned}
$$

$$
\begin{aligned}
y_{(3 k+3)(\sigma+1)+2 k+4} & =\frac{\alpha_{13} z_{(3 k+3)(\sigma+1)+k+3}}{\alpha_{14}+\alpha_{15} \prod_{i=0}^{k} x_{(3 k+3) \sigma+5 k+6-i}}, \\
& \leq \frac{\alpha_{13}}{\alpha_{14}} z_{(3 k+3)(\sigma+1)+k+3} \leq\left(\frac{\alpha_{10}}{\alpha_{11}} \frac{\alpha_{13}}{\alpha_{14}} \frac{\alpha_{16}}{\alpha_{17}}\right)^{\sigma+2} y_{-k+1}, \\
y_{(3 k+3)(\sigma+1)+3 k+2} & =\frac{\alpha_{13} z_{(3 k+3)(\sigma+1)+2 k+1}}{\alpha_{14}+\alpha_{15} \prod_{i=0}^{k} x_{(3 k+3) \sigma+6 k+4-i}}, \\
& \leq \frac{\alpha_{13}}{\alpha_{14}} z_{(3 k+3)(\sigma+1)+2 k+1} \leq\left(\frac{\alpha_{10}}{\alpha_{11}} \frac{\alpha_{13}}{\alpha_{14}} \frac{\alpha_{16}}{\alpha_{17}}\right)^{\sigma+2} y_{-1}, \\
y_{(3 k+3)(\sigma+1)+3 k+3} & =\frac{\alpha_{13} z_{(3 k+3)(\sigma+1)+2 k+2}}{\alpha_{14}+\alpha_{15} \prod_{i=0}^{k} x_{(3 k+3) \sigma+6 k+5-i}}, \\
& \leq \frac{\alpha_{13}}{\alpha_{14}} z_{(3 k+3)(\sigma+1)+2 k+2} \leq\left(\frac{\alpha_{10}}{\alpha_{11}} \frac{\alpha_{13}}{\alpha_{14}} \frac{\alpha_{16}}{\alpha_{17}}\right)^{\sigma+2} y_{0},
\end{aligned}
$$$$
\begin{aligned}
z_{(3 k+3)(\sigma+1)+2 k+3} & =\frac{\alpha_{16} x_{(3 k+3)(\sigma+1)+k+2}}{\alpha_{17}+\alpha_{18} \prod_{i=0}^{k} y_{(3 k+3) \sigma+5 k+5-i}} \\
& \leq \frac{\alpha_{16}}{\alpha_{17}} x_{(3 k+3)(\sigma+1)+k+2} \leq\left(\frac{\alpha_{10}}{\alpha_{11}} \frac{\alpha_{13}}{\alpha_{14}} \frac{\alpha_{16}}{\alpha_{17}}\right)^{\sigma+2} z_{-k},
\end{aligned}
$$$$
z_{(3 k+3)(\sigma+1)+2 k+4}=\frac{\alpha_{16} x_{(3 k+3)(\sigma+1)+k+3}}{\alpha_{17}+\alpha_{18} \prod_{i=0}^{k} y_{(3 k+3) \sigma+5 k+6-i}},
$$$$
\leq \frac{\alpha_{16}}{\alpha_{17}} x_{(3 k+3)(\sigma+1)+k+3} \leq\left(\frac{\alpha_{10}}{\alpha_{11}} \frac{\alpha_{13}}{\alpha_{14}} \frac{\alpha_{16}}{\alpha_{17}}\right)^{\sigma+2} z_{-k+1} \text {, }
$$$$
\begin{aligned}
z_{(3 k+3)(\sigma+1)+3 k+2} & =\frac{\alpha_{16} x_{(3 k+3)(\sigma+1)+2 k+1}}{\alpha_{17}+\alpha_{18} \prod_{i=0}^{k} y_{(3 k+3) \sigma+6 k+4-i}} \\
& \leq \frac{\alpha_{16}}{\alpha_{17}} x_{(3 k+3)(\sigma+1)+2 k+1} \leq\left(\frac{\alpha_{10}}{\alpha_{11}} \frac{\alpha_{13}}{\alpha_{14}} \frac{\alpha_{16}}{\alpha_{17}}\right)^{\sigma+2} z_{-1},
\end{aligned}
$$$$
z_{(3 k+3)(\sigma+1)+3 k+3}=\frac{\alpha_{16} x_{(3 k+3)(\sigma+1)+2 k+2}}{\alpha_{17}+\alpha_{18} \prod_{i=0}^{k} y_{(3 k+3) \sigma+6 k+5-i}},
$$$$
\leq \frac{\alpha_{16}}{\alpha_{17}} x_{(3 k+3)(\sigma+1)+2 k+2} \leq\left(\frac{\alpha_{10}}{\alpha_{11}} \frac{\alpha_{13}}{\alpha_{14}} \frac{\alpha_{16}}{\alpha_{17}}\right)^{\sigma+2} z_{0} \text {. }
$$

Corollary 4. $\left\{X_{n}\right\}$ of (11) is bounded if (30) holds.

Proof. It is a direct result of Theorem 3.

Hereafter, we will present boundedness for discrete systems (12)-(14). 
Theorem 5. (i) If $\left\{X_{n}\right\}$ is the positive solution of (12), then for $\mu \geq 0$, the following holds:

$$
\begin{aligned}
& \begin{cases}\left(\frac{\alpha_{19}}{\alpha_{20}}\right)^{\mu+1}\left(\frac{\alpha_{22}}{\alpha_{23}}\right)^{\mu}\left(\frac{\alpha_{25}}{\alpha_{26}}\right)^{\mu} z_{-k}, & \text { if } n=(3+3 k) \mu+1, \\
\left(\frac{\alpha_{19}}{\alpha_{20}}\right)^{\mu+1}\left(\frac{\alpha_{22}}{\alpha_{23}}\right)^{\mu}\left(\frac{\alpha_{25}}{\alpha_{26}}\right)^{\mu} z_{-k+1}, & \text { if } n=(3+3 k) \mu+2,\end{cases} \\
& \text { : } \\
& \left(\frac{\alpha_{19}}{\alpha_{20}}\right)^{\mu+1}\left(\frac{\alpha_{22}}{\alpha_{23}}\right)^{\mu}\left(\frac{\alpha_{25}}{\alpha_{26}}\right)^{\mu} z_{-1}, \quad \text { if } n=(3+3 k) \mu+k, \\
& \left(\frac{\alpha_{19}}{\alpha_{20}}\right)^{\mu+1}\left(\frac{\alpha_{22}}{\alpha_{23}}\right)^{\mu}\left(\frac{\alpha_{25}}{\alpha_{26}}\right)^{\mu} z_{0}, \quad \text { if } n=(3+3 k) \mu+k+1, \\
& \left(\frac{\alpha_{19}}{\alpha_{20}}\right)^{\mu+1}\left(\frac{\alpha_{22}}{\alpha_{23}}\right)^{\mu+1}\left(\frac{\alpha_{25}}{\alpha_{26}}\right)^{\mu} y_{-k}, \quad \text { if } n=(3+3 k) \mu+k+2 \text {, } \\
& \left(\frac{\alpha_{19}}{\alpha_{20}}\right)^{\mu+1}\left(\frac{\alpha_{22}}{\alpha_{23}}\right)^{\mu+1}\left(\frac{\alpha_{25}}{\alpha_{26}}\right)^{\mu} y_{-k+1}, \quad \text { if } n=(3+3 k) \mu+k+3, \\
& 0<x_{n} \leq \quad \vdots \\
& \left(\frac{\alpha_{19}}{\alpha_{20}}\right)^{\mu+1}\left(\frac{\alpha_{22}}{\alpha_{23}}\right)^{\mu+1}\left(\frac{\alpha_{25}}{\alpha_{26}}\right)^{\mu} y_{-1}, \quad \text { if } n=(3+3 k) \mu+2 k+1, \\
& \left(\left(\frac{\alpha_{19}}{\alpha_{20}}\right)^{\mu+1}\left(\frac{\alpha_{22}}{\alpha_{23}}\right)^{\mu+1}\left(\frac{\alpha_{25}}{\alpha_{26}}\right)^{\mu} y_{0}, \quad \text { if } n=(3+3 k) \mu+2 k+2\right. \text {, } \\
& \left(\frac{\alpha_{19}}{\alpha_{20}} \frac{\alpha_{22}}{\alpha_{23}} \frac{\alpha_{25}}{\alpha_{26}}\right)^{\mu+1} x_{-k}, \quad \text { if } n=(3+3 k) \mu+2 k+3 \text {, } \\
& \left(\frac{\alpha_{19}}{\alpha_{20}} \frac{\alpha_{22}}{\alpha_{23}} \frac{\alpha_{25}}{\alpha_{26}}\right)^{\mu+1} x_{-k+1}, \quad \text { if } n=(3+3 k) \mu+2 k+3 \text {, } \\
& \text { : } \\
& \begin{array}{ll}
\left(\frac{\alpha_{19}}{\alpha_{20}} \frac{\alpha_{22}}{\alpha_{23}} \frac{\alpha_{25}}{\alpha_{26}}\right)^{\mu+1} x_{-1}, & \text { if } n=(3+3 k) \mu+3 k+2, \\
\left(\frac{\alpha_{19}}{\alpha_{20}} \frac{\alpha_{22}}{\alpha_{23}} \frac{\alpha_{25}}{\alpha_{26}}\right)^{\mu+1} x_{0}, & \text { if } n=(3+3 k) \mu+3 k+3 .
\end{array} \\
& \begin{cases}\left(\frac{\alpha_{19}}{\alpha_{20}}\right)^{\mu}\left(\frac{\alpha_{22}}{\alpha_{23}}\right)^{\mu+1}\left(\frac{\alpha_{25}}{\alpha_{26}}\right)^{\mu} x_{-k}, \quad \text { if } n=(3+3 k) \mu+1, \\
\left(\frac{\alpha_{19}}{\alpha_{20}}\right)^{\mu}\left(\frac{\alpha_{22}}{\alpha_{23}}\right)^{\mu+1}\left(\frac{\alpha_{25}}{\alpha_{26}}\right)^{\mu} x_{-k+1}, & \text { if } n=(3+3 k) \mu+2,\end{cases} \\
& \text { ! } \\
& \left(\frac{\alpha_{19}}{\alpha_{20}}\right)^{\mu}\left(\frac{\alpha_{22}}{\alpha_{23}}\right)^{\mu+1}\left(\frac{\alpha_{25}}{\alpha_{26}}\right)^{\mu} x_{-1}, \quad \text { if } n=(3+3 k) \mu+k, \\
& \left(\frac{\alpha_{19}}{\alpha_{20}}\right)^{\mu}\left(\frac{\alpha_{22}}{\alpha_{23}}\right)^{\mu+1}\left(\frac{\alpha_{25}}{\alpha_{26}}\right)^{\mu} x_{0}, \quad \text { if } n=(3+3 k) \mu+k+1, \\
& \left(\frac{\alpha_{19}}{\alpha_{20}}\right)^{\mu}\left(\frac{\alpha_{22}}{\alpha_{23}}\right)^{\mu+1}\left(\frac{\alpha_{25}}{\alpha_{26}}\right)^{\mu} z_{-k}, \quad \text { if } n=(3+3 k) \mu+k+2 \text {, } \\
& \left(\frac{\alpha_{19}}{\alpha_{20}}\right)^{\mu}\left(\frac{\alpha_{22}}{\alpha_{23}}\right)^{\mu+1}\left(\frac{\alpha_{25}}{\alpha_{26}}\right)^{\mu} z_{-k+1} \text {, if } n=(3+3 k) \mu+k+3, \\
& 0<y_{n} \leq \quad \vdots \\
& \left(\frac{\alpha_{19}}{\alpha_{20}}\right)^{\mu}\left(\frac{\alpha_{22}}{\alpha_{23}}\right)^{\mu+1}\left(\frac{\alpha_{25}}{\alpha_{26}}\right)^{\mu} z_{-1}, \quad \text { if } n=(3+3 k) \mu+2 k+1 \text {, } \\
& \left(\frac{\alpha_{19}}{\alpha_{20}}\right)^{\mu}\left(\frac{\alpha_{22}}{\alpha_{23}}\right)^{\mu+1}\left(\frac{\alpha_{25}}{\alpha_{26}}\right)^{\mu} z_{0}, \quad \text { if } n=(3+3 k) \mu+2 k+2, \\
& \left(\frac{\alpha_{19}}{\alpha_{20}} \frac{\alpha_{22}}{\alpha_{23}} \frac{\alpha_{25}}{\alpha_{26}}\right)^{\mu+1} y_{-k}, \quad \text { if } n=(3 k+3) \mu+2 k+3 \text {, } \\
& \left(\frac{\alpha_{19}}{\alpha_{20}} \frac{\alpha_{22}}{\alpha_{23}} \frac{\alpha_{25}}{\alpha_{26}}\right)^{\mu+1} y_{-k+1}, \quad \text { if } n=(3+3 k) \mu+2 k+3 \text {, } \\
& \vdots \\
& \begin{array}{ll}
\left(\frac{\alpha_{19}}{\alpha_{20}} \frac{\alpha_{22}}{\alpha_{23}} \frac{\alpha_{25}}{\alpha_{26}}\right)^{\mu+1} y_{-1}, & \text { if } n=(3+3 k) \mu+3 k+2, \\
\left(\frac{\alpha_{19}}{\alpha_{20}} \frac{\alpha_{22}}{\alpha_{23}} \frac{\alpha_{25}}{\alpha_{26}}\right)^{\mu+1} y_{0}, & \text { if } n=(3+3 k) \mu+3 k+3 .
\end{array}
\end{aligned}
$$

Finally,

$$
\begin{aligned}
& \begin{cases}\left(\frac{\alpha_{19}}{\alpha_{20}}\right)^{\mu}\left(\frac{\alpha_{22}}{\alpha_{23}}\right)^{\mu}\left(\frac{\alpha_{25}}{\alpha_{26}}\right)^{\mu+1} y_{-k}, & \text { if } n=(3+3 k) \mu+1, \\
\left(\frac{\alpha_{19}}{\alpha_{20}}\right)^{\mu}\left(\frac{\alpha_{22}}{\alpha_{23}}\right)^{\mu}\left(\frac{\alpha_{25}}{\alpha_{26}}\right)^{\mu+1} y_{-k+1}, & \text { if } n=(3+3 k) \mu+2,\end{cases} \\
& \left(\frac{\alpha_{19}}{\alpha_{20}}\right)^{\mu}\left(\frac{\alpha_{22}}{\alpha_{23}}\right)^{\mu}\left(\frac{\alpha_{25}}{\alpha_{26}}\right)^{\mu+1} y_{-1}, \quad \text { if } n=(3+3 k) \mu+k, \\
& \left(\frac{\alpha_{19}}{\alpha_{20}}\right)^{\mu}\left(\frac{\alpha_{22}}{\alpha_{23}}\right)^{\mu}\left(\frac{\alpha_{25}}{\alpha_{26}}\right)^{\mu+1} y_{0}, \quad \text { if } n=(3+3 k) \mu+k+1, \\
& \left(\frac{\alpha_{19}}{\alpha_{20}}\right)^{\mu+1}\left(\frac{\alpha_{22}}{\alpha_{23}}\right)^{\mu}\left(\frac{\alpha_{25}}{\alpha_{26}}\right)^{\mu+1} x_{-k}, \quad \text { if } n=(3+3 k) \mu+k+2, \\
& \left(\frac{\alpha_{19}}{\alpha_{20}}\right)^{\mu+1}\left(\frac{\alpha_{22}}{\alpha_{23}}\right)^{\mu}\left(\frac{\alpha_{25}}{\alpha_{26}}\right)^{\mu+1} x_{-k+1}, \quad \text { if } n=(3+3 k) \mu+k+3, \\
& 0<z_{n} \leq \quad \vdots \\
& \left(\frac{\alpha_{19}}{\alpha_{20}}\right)^{\mu+1}\left(\frac{\alpha_{22}}{\alpha_{23}}\right)^{\mu}\left(\frac{\alpha_{25}}{\alpha_{26}}\right)^{\mu+1} x_{-1}, \quad \text { if } n=(3+3 k) \mu+2 k+1 \text {, } \\
& \left(\frac{\alpha_{19}}{\alpha_{20}}\right)^{\mu+1}\left(\frac{\alpha_{22}}{\alpha_{23}}\right)^{\mu}\left(\frac{\alpha_{25}}{\alpha_{26}}\right)^{\mu+1} x_{0}, \quad \text { if } n=(3+3 k) \mu+2 k+2, \\
& \left(\frac{\alpha_{19}}{\alpha_{20}} \frac{\alpha_{22}}{\alpha_{23}} \frac{\alpha_{25}}{\alpha_{26}}\right)^{\mu+1} z_{-k}, \quad \text { if } n=(3+3 k) \mu+2 k+3 \text {, } \\
& \left(\frac{\alpha_{19}}{\alpha_{20}} \frac{\alpha_{22}}{\alpha_{23}} \frac{\alpha_{25}}{\alpha_{26}}\right)^{\mu+1} z_{-k+1}, \quad \text { if } n=(3+3 k) \mu+2 k+3 \text {, } \\
& \vdots \\
& \begin{array}{ll}
\left(\frac{\alpha_{19}}{\alpha_{20}} \frac{\alpha_{22}}{\alpha_{23}} \frac{\alpha_{25}}{\alpha_{26}}\right)^{\mu+1} z_{-1}, & \text { if } n=(3+3 k) \mu+3 k+2, \\
\left(\frac{\alpha_{19}}{\alpha_{20}} \frac{\alpha_{22}}{\alpha_{23}} \frac{\alpha_{25}}{\alpha_{26}}\right)^{\mu+1} z_{0}, & \text { if } n=(3+3 k) \mu+3 k+3 .
\end{array}
\end{aligned}
$$

(ii) If $\left\{X_{n}\right\}$ is the positive solution of (13), then for $\mu \geq 0$, the following holds:

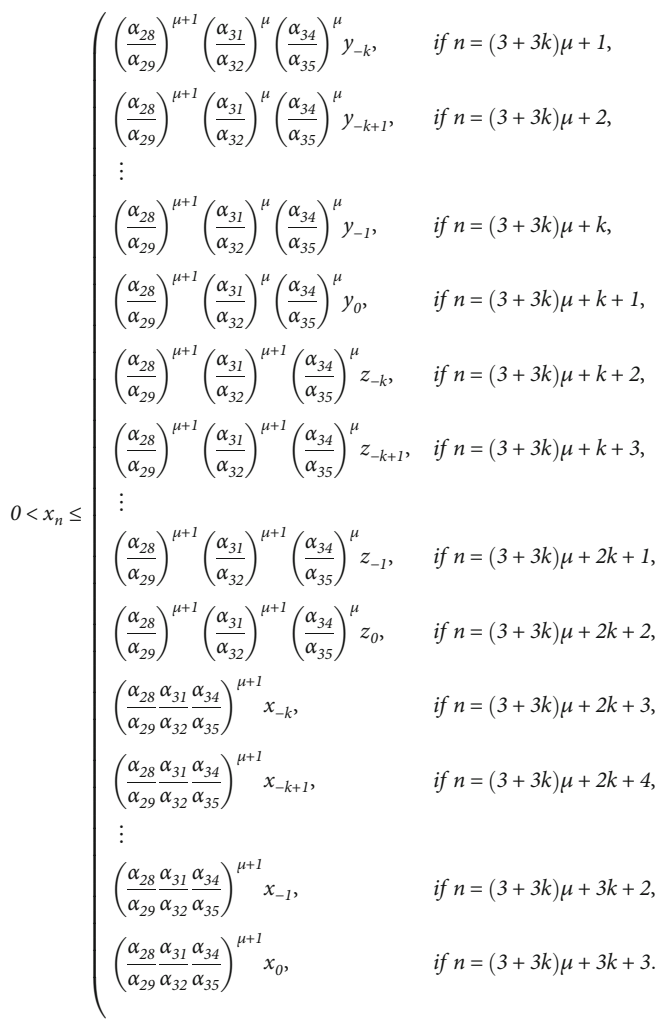


And

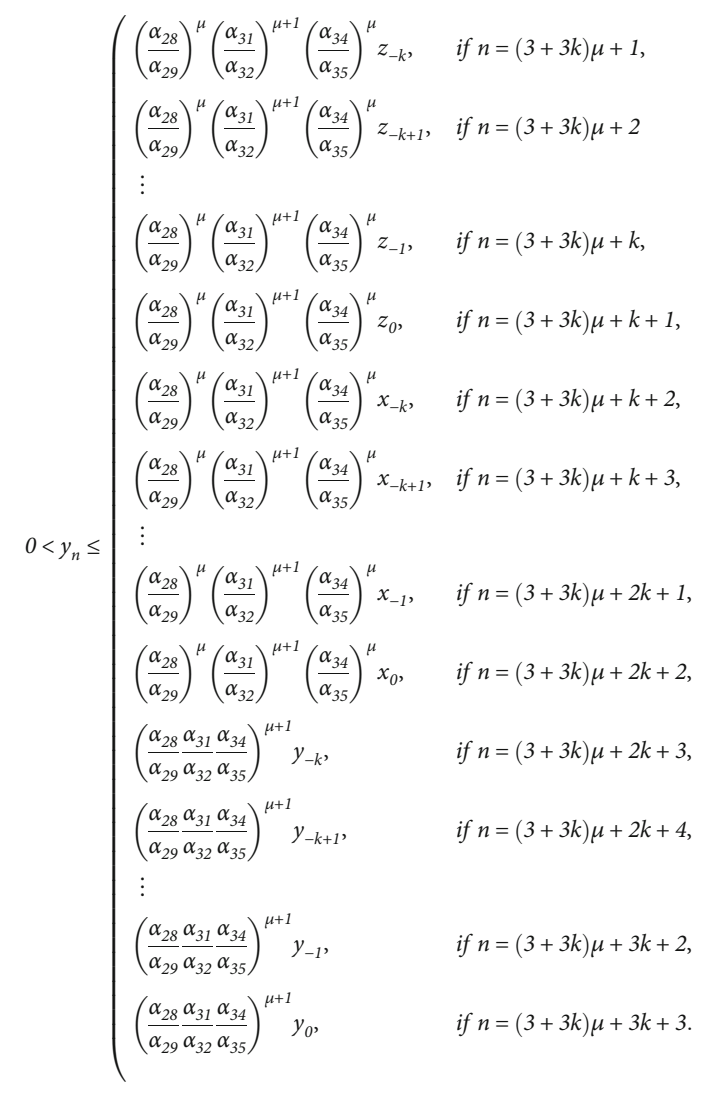

(iii) If $\left\{X_{n}\right\}$ is the positive solution of (14), then for $\mu \geq 0$, the following holds:

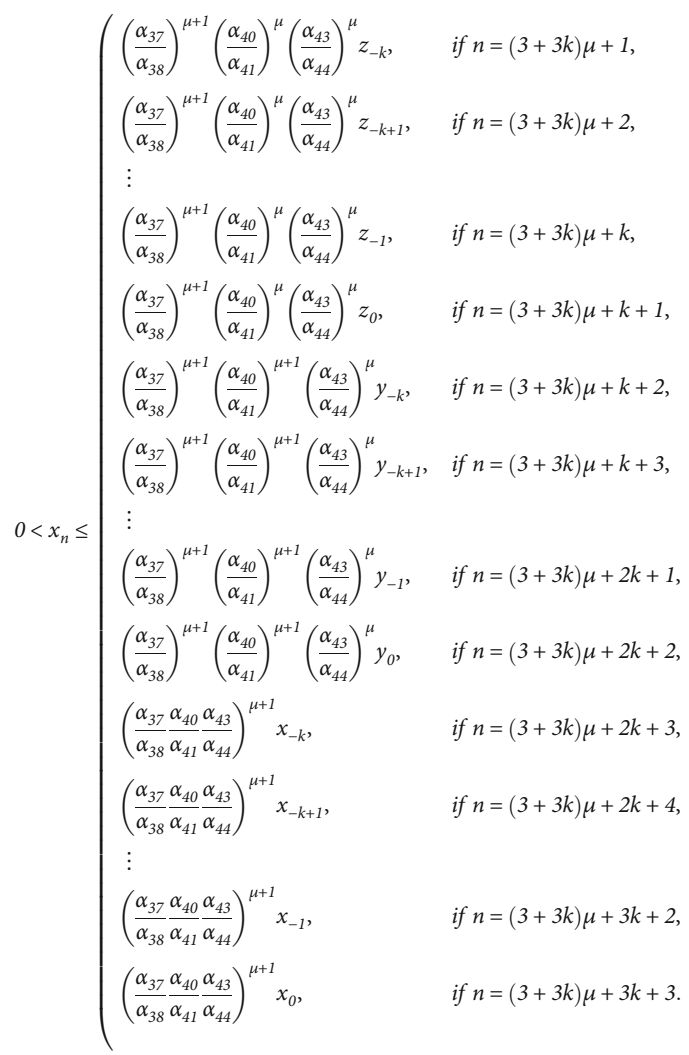

And

$$
\left\{\begin{array}{lll}
\left(\frac{\alpha_{37}}{\alpha_{38}}\right)^{\mu}\left(\frac{\alpha_{40}}{\alpha_{41}}\right)^{\mu+1}\left(\frac{\alpha_{43}}{\alpha_{44}}\right)^{\mu} x_{-k}, & \text { if } n=(3+3 k) \mu+1, \\
\left(\frac{\alpha_{37}}{\alpha_{38}}\right)^{\mu}\left(\frac{\alpha_{40}}{\alpha_{41}}\right)^{\mu+1}\left(\frac{\alpha_{43}}{\alpha_{44}}\right)^{\mu} x_{-k+1}, & \text { if } n=(3+3 k) \mu+2, \\
\vdots \\
\left(\frac{\alpha_{37}}{\alpha_{38}}\right)^{\mu}\left(\frac{\alpha_{40}}{\alpha_{41}}\right)^{\mu+1}\left(\frac{\alpha_{43}}{\alpha_{44}}\right)^{\mu} x_{-1}, & \text { if } n=(3+3 k) \mu+k, \\
\left(\frac{\alpha_{37}}{\alpha_{38}}\right)^{\mu}\left(\frac{\alpha_{40}}{\alpha_{41}}\right)^{\mu+1}\left(\frac{\alpha_{43}}{\alpha_{44}}\right)^{\mu} x_{0}, & \text { if } n=(3+3 k) \mu+k+1, \\
\left(\frac{\alpha_{37}}{\alpha_{38}}\right)^{\mu}\left(\frac{\alpha_{40}}{\alpha_{41}}\right)^{\mu+1}\left(\frac{\alpha_{43}}{\alpha_{44}}\right)^{\mu} z_{-k}, & \text { if } n=(3+3 k) \mu+k+2, \\
\left(\frac{\alpha_{37}}{\alpha_{38}}\right)^{\mu}\left(\frac{\alpha_{40}}{\alpha_{41}}\right)^{\mu+1}\left(\frac{\alpha_{43}}{\alpha_{44}}\right)^{\mu} z_{-k+1}, & \text { if } n=(3+3 k) \mu+k+3, \\
\vdots & \\
\left(\frac{\alpha_{37}}{\alpha_{38}}\right)^{\mu}\left(\frac{\alpha_{40}}{\alpha_{41}}\right)^{\mu+1}\left(\frac{\alpha_{43}}{\alpha_{44}}\right)^{\mu} z_{-1}, & \text { if } n=(3+3 k) \mu+2 k+1, \\
\left(\frac{\alpha_{37}}{\alpha_{38}}\right)^{\mu}\left(\frac{\alpha_{40}}{\alpha_{41}}\right)^{\mu+1}\left(\frac{\alpha_{43}}{\alpha_{44}}\right)^{\mu} z_{0}, & \text { if } n=(3+3 k) \mu+2 k+2, \\
\left(\frac{\alpha_{37}}{\alpha_{38}} \frac{\alpha_{40}}{\alpha_{41}} \frac{\alpha_{43}}{\alpha_{44}}\right)^{\mu+1} y_{-k}, & \text { if } n=(3+3 k) \mu+2 k+3, \\
\left(\frac{\alpha_{37}}{\alpha_{38}} \frac{\alpha_{40}}{\alpha_{41}} \frac{\alpha_{43}}{\alpha_{44}}\right)^{\mu+1} y_{-k+1}, & \text { if } n=(3+3 k) \mu+2 k+4, \\
\vdots \\
\left(\frac{\alpha_{37}}{\alpha_{38}} \frac{\alpha_{40}}{\alpha_{41}} \frac{\alpha_{43}}{\alpha_{44}}\right)^{\mu+1} y_{-1}, & \text { if } n=(3+3 k) \mu+3 k+2, \\
\left(\frac{\alpha_{37}}{\alpha_{38}} \frac{\alpha_{40}}{\alpha_{41}} \frac{\alpha_{43}}{\alpha_{44}}\right)^{m+1} y_{0}, & \\
&
\end{array}\right.
$$


Finally,

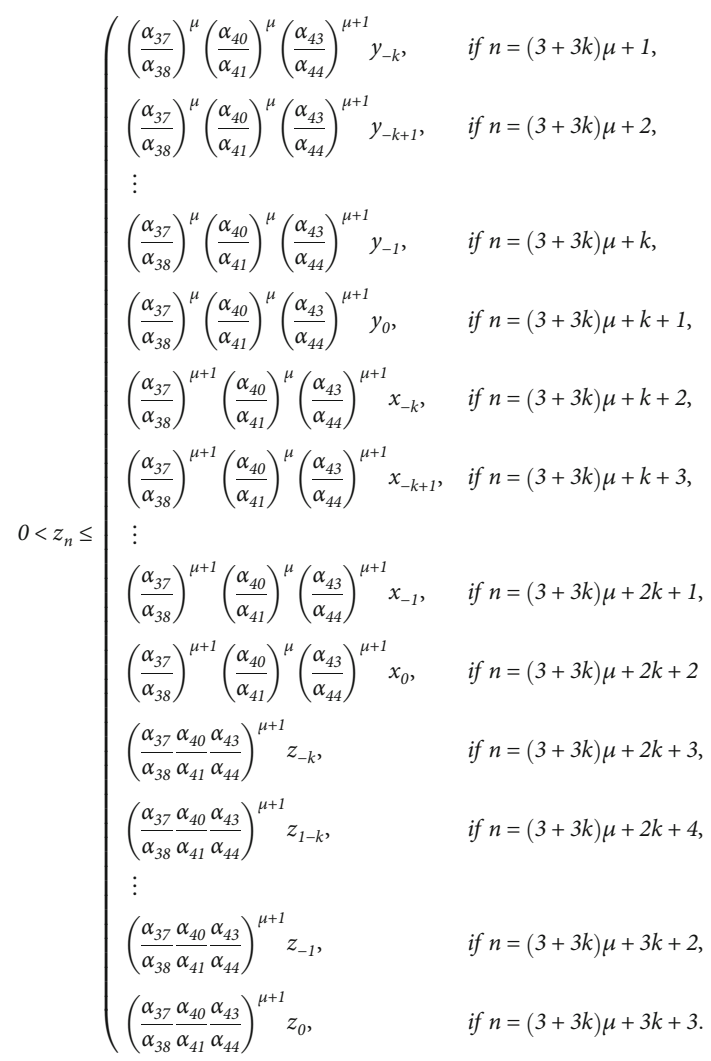

Proof. Same as the proof of Theorem 3.

Corollary 6. The following statements are true for (12)-(14):

(i) If (40) holds, then $\left\{\left(X_{n}\right)\right\}$ of discrete system (12) is bounded

(ii) If (41) holds, then $\left\{\left(X_{n}\right)\right\}$ of discrete system (13) is bounded

(iii) If (42) holds, then $\left\{\left(X_{n}\right)\right\}$ of discrete system (14) is bounded

Proof. Its consequence of Theorem 5.

\section{Global Dynamics about $P_{0}$ of (11)-(14)}

Theorem 7. If (30) holds, then $P_{0}$ of (11) is globally stable.

Proof. If (30) is true, then from discrete system (11), one has

$$
\begin{aligned}
& x_{n+1}=\frac{\alpha_{10} y_{n-k}}{\alpha_{11}+\alpha_{12} \prod_{i=0}^{k} z_{n-i}} \leq \frac{\alpha_{10}}{\alpha_{11}} y_{n-k}<y_{n-k}, \\
& y_{n+1}=\frac{\alpha_{13} z_{n-k}}{\alpha_{14}+\alpha_{15} \prod_{i=0}^{k} x_{n-i}} \leq \frac{\alpha_{13}}{\alpha_{14}} z_{n-k}<z_{n-k}, \\
& z_{n+1}=\frac{\alpha_{16} x_{n-k}}{\alpha_{17}+\alpha_{18} \prod_{i=0}^{k} y_{n-i}} \leq \frac{\alpha_{16}}{\alpha_{17}} x_{n-1}<x_{n-k} .
\end{aligned}
$$

From (59), one gets

$$
\begin{aligned}
& x_{(3 k+3) n+1}<y_{(3 k+3) n-k} \text { and } x_{(3 k+3) n+3 k+4}<y_{(3 k+3) n+2 k+3}, \\
& y_{(3 k+3) n+1}<z_{(3 k+3)-k} \text { and } y_{(3 k+3) n+3 k+4}<z_{(3 k+3) n+2 k+3}, \\
& z_{(3 k+3) n+1}<x_{(3 k+3) n-k} \text { and } z_{(3 k+3) n+3 k+4}<x_{(3 k+3) n+2 k+3} .
\end{aligned}
$$

Moreover, from (60), one gets

$$
\begin{aligned}
& x_{(3 k+3) n+3 k+4}<y_{(3 k+3) n+2 k+3}<z_{(3 k+3) n+k+2}<x_{(3 k+3) n+1}, \\
& y_{(3 k+3) n+3 k+4}<z_{(3 k+3) n+2 k+3}<x_{(3 k+3) n+k+2}<y_{(3 k+3) n+1}, \\
& z_{(3 k+3) n+3 k+4}<x_{(3 k+3) n+2 k+3}<y_{(3 k+3) n+k+2}<z_{(3 k+3) n+1}
\end{aligned}
$$

From (61), one can conclude that $\left\{x_{(3 k+3) n+1}\right\}, \cdots,\{$ $\left.x_{(3 k+3) n+3 k+3}\right\} ; \quad\left\{y_{(3 k+3) n+1}\right\}, \cdots,\left\{y_{(3 k+3) n+3 k+3}\right\} ; \quad$ and \{ $\left.z_{(3 k+3) n+1}\right\}, \cdots,\left\{z_{(3 k+3) n+3 k+3}\right\}$ are decreasing. Thus, $\left\{x_{n}\right\},\{$ $\left.y_{n}\right\}$, and $\left\{z_{n}\right\}$ are decreasing, and hence, one can conclude that $\lim _{n \longrightarrow \infty}\left(x_{n}, y_{n}, z_{n}\right)=P_{0}$.

Theorem 8. If respective parametric conditions (40), (41), and (42) hold, then $P_{0}$ of discrete systems (12)-(14) is globally stable.

Proof. Like as the proof of Theorem 7.

\section{Convergence Rate}

Theorem 9. If respective parametric conditions (30), (40), (41), and (42) hold, then error vector

$$
\widetilde{\mathrm{\varrho}_{n}}=\left(\begin{array}{c}
\mathrm{\varrho}_{n}^{1} \\
\vdots \\
\mathrm{\varrho}_{n-k}^{1} \\
\mathrm{\varrho}_{n}^{2} \\
\vdots \\
\mathrm{\varrho}_{n-k}^{2} \\
\mathrm{\varrho}_{n}^{3} \\
\vdots \\
\mathrm{\varrho}_{n-k}^{3}
\end{array}\right),
$$

of positive solution: $\left\{X_{n}\right\}$ of corresponding discrete systems (11)-(14) satisfies the following relations: 


$$
\begin{aligned}
& \lim _{n \rightarrow \infty} \sqrt[n]{\left\|\mathrm{Q}_{n}\right\|}=|\nu J|_{P_{0}} \mid, \\
& \lim _{n \longrightarrow \infty} \frac{\left\|\mathrm{Q}_{n+1}\right\|}{\left\|\mathrm{Q}_{n}\right\|}=|v J|_{P_{0}} \mid,
\end{aligned}
$$

where $|v J|_{P_{0}} \mid$ is equivalent to the modulus of one of the characteristic roots of $\left.J\right|_{P_{0}}$ calculated at trivial fixed point $P_{0}$.

Proof. Let $\left\{\left(X_{n}\right)\right\}$ be a positive solution of (11) for which $\lim _{n \longrightarrow \infty}\left(x_{n}, y_{n}, z_{n}\right)=\Lambda$. In order for error terms, we have

$$
\begin{aligned}
& x_{n+1}-x=\frac{\alpha_{10} y_{n-k}}{\alpha_{11}+\alpha_{12} \prod_{i=0}^{k} z_{n-i}}-\frac{\alpha_{10} y}{\alpha_{11}+\alpha_{12} z^{k+1}}, \\
& =\frac{\alpha_{10}}{\alpha_{11}+\alpha_{12} \prod_{i=0}^{k} z_{n-i}}\left(y_{n-k}-y\right) \\
& -\frac{\alpha_{10} \alpha_{12} y \prod_{i=1}^{k} z_{n-i}}{\left(\alpha_{11}+\alpha_{12} \prod_{i=0}^{k} z_{n-i}\right)\left(\alpha_{11}+\alpha_{12} z^{k+1}\right)}\left(z_{n}-z\right) \\
& -\frac{\alpha_{10} \alpha_{12} y z \prod_{i=2}^{k} z_{n-i}}{\left(\alpha_{11}+\alpha_{12} \prod_{i=0}^{k} z_{n-i}\right)\left(\alpha_{11}+\alpha_{12} z^{k+1}\right)}\left(z_{n-1}-z\right) \\
& -\cdots-\frac{\alpha_{10} \alpha_{12} y z^{k-1} z_{n-k}}{\left(\alpha_{11}+\alpha_{12} \prod_{i=0}^{k} z_{n-i}\right)\left(\alpha_{11}+\alpha_{12} z^{k+1}\right)}\left(z_{n-k+1}-z\right) \\
& -\frac{\alpha_{10} \alpha_{12} y z^{k}}{\left(\alpha_{11}+\alpha_{12} \prod_{i=0}^{k} z_{n-i}\right)\left(\alpha_{11}+\alpha_{12} z^{k+1}\right)}\left(z_{n-k}-z\right), \\
& y_{n+1}-y=\frac{\alpha_{13} z_{n-k}}{\alpha_{14}+\alpha_{15} \prod_{i=0}^{k} x_{n-i}}-\frac{\alpha_{13} z}{\alpha_{14}+\alpha_{15} x^{k+1}}, \\
& =-\frac{\alpha_{13} \alpha_{15} z \prod_{i=1}^{k} x_{n-i}}{\left(\alpha_{14}+\alpha_{15} \prod_{i=0}^{k} x_{n-i}\right)\left(\alpha_{14}+\alpha_{15} x^{k+1}\right)}\left(x_{n}-x\right) \\
& -\frac{\alpha_{13} \alpha_{15} z x \prod_{i=2}^{k} x_{n-i}}{\left(\alpha_{14}+\alpha_{15} \prod_{i=0}^{k} x_{n-i}\right)\left(\alpha_{14}+\alpha_{15} x^{k+1}\right)}\left(x_{n-1}-x\right) \\
& -\cdots-\frac{\alpha_{13} \alpha_{15} z x^{k-1} x_{n-k}}{\left(\alpha_{14}+\alpha_{15} \prod_{i=0}^{k} x_{n-i}\right)\left(\alpha_{14}+\alpha_{15} x^{k+1}\right)}\left(x_{n-k+1}-x\right) \\
& -\frac{\alpha_{13} \alpha_{15} z x^{k}}{\left(\alpha_{14}+\alpha_{15} \prod_{i=0}^{k} x_{n-i}\right)\left(\alpha_{14}+\alpha_{15} x^{k+1}\right)}\left(x_{n-k}-x\right) \\
& +\frac{\alpha_{13}}{\alpha_{14}+\alpha_{15} \prod_{i=0}^{1} x_{n-k}}\left(z_{n-k}-z\right) \text {, } \\
& z_{n+1}-z=\frac{\alpha_{16} x_{n-k}}{\alpha_{17}+\alpha_{18} \prod_{i=0}^{k} y_{n-i}}-\frac{\alpha_{16} x}{\alpha_{17}+\alpha_{18} y^{k+1}}, \\
& =\frac{\alpha_{16}}{\alpha_{17}+\alpha_{18} \prod_{i=0}^{k} y_{n-i}}\left(x_{n-k}-x\right) \\
& -\frac{\alpha_{16} \alpha_{18} x \prod_{i=1}^{k} y_{n-i}}{\left(\alpha_{17}+\alpha_{18} \prod_{i=0}^{k} y_{n-i}\right)\left(\alpha_{17}+\alpha_{18} y^{k+1}\right)}\left(y_{n}-y\right) \\
& -\frac{\alpha_{16} \alpha_{18} x y \prod_{i=2}^{k} y_{n-i}}{\left(\alpha_{17}+\alpha_{18} \prod_{i=0}^{k} y_{n-i}\right)\left(\alpha_{17}+\alpha_{18} y^{k+1}\right)}\left(y_{n-1}-y\right) \\
& -\cdots-\frac{\alpha_{16} \alpha_{18} x y^{k-1} y_{n-k}}{\left(\alpha_{17}+\alpha_{18} \prod_{i=0}^{k} y_{n-i}\right)\left(\alpha_{17}+\alpha_{18} y^{k+1}\right)}\left(y_{n-k+1}-y\right) \\
& \cdot \frac{\alpha_{16} \alpha_{18} x y^{k}}{\left(\alpha_{17}+\alpha_{18} \prod_{i=0}^{k} y_{n-i}\right)\left(\alpha_{17}+\alpha_{18} y^{k+1}\right)}\left(y_{n-k}-y\right) \text {. }
\end{aligned}
$$

Now, set

$$
\begin{aligned}
& \mathrm{\varrho}_{n}^{1}=x_{n}-x, \\
& \mathrm{\varrho}_{n}^{2}=y_{n}-y, \\
& \mathrm{\varrho}_{n}^{3}=z_{n}-z .
\end{aligned}
$$

Utilizing (67) in (64) and (66), one gets

$$
\begin{aligned}
& \mathrm{Q}_{n+1}^{1}=A_{n}^{n-k} \mathrm{e}_{n-k}^{2}+B_{n}^{n} \mathrm{e}_{n}^{3}+B_{n}^{n-1} \mathrm{e}_{n-1}^{3}+\cdots+B_{n}^{n-k+1} \mathrm{e}_{n-k+1}^{3}+B_{n}^{n-k} \mathrm{e}_{n-k}^{3}, \\
& \mathrm{Q}_{n+1}^{2}=C_{n}^{n} \mathrm{e}_{n}^{1}+C_{n}^{n-1} \mathrm{e}_{n-1}^{1}+\cdots+C_{n}^{n-k+1} \mathrm{e}_{n-k+1}^{1}+C_{n}^{n-k} \mathrm{e}_{n-k}^{1}+D_{n}^{n-k} \mathrm{e}_{n-k}^{3}, \\
& \mathrm{Q}_{n+1}^{3}=E_{n}^{n-k} \mathrm{e}_{n-k}^{1}+F_{n}^{n} \mathrm{Q}_{n}^{2}+F_{n}^{n-1} \mathrm{e}_{n-1}^{2}+\cdots+F_{n}^{n-k+1} \mathrm{e}_{n-k+1}^{2}+F_{n}^{n-k} \mathrm{e}_{n-k}^{2},
\end{aligned}
$$

where

$$
\begin{aligned}
& A_{n}^{n-k}=\frac{\alpha_{10}}{\alpha_{11}+\alpha_{12} z^{k+1}}, \\
& B_{n}^{n}=-\frac{\alpha_{10} \alpha_{12} y \prod_{i=1}^{k} z_{n-i}}{\left(\alpha_{11}+\alpha_{12} \prod_{i=0}^{k} z_{n-i}\right)\left(\alpha_{11}+\alpha_{12} z^{k+1}\right)}, \\
& B_{n}^{n-1}=-\frac{\alpha_{10} \alpha_{12} y z \prod_{i=2}^{k} z_{n-i}}{\left(\alpha_{11}+\alpha_{12} \prod_{i=0}^{k} z_{n-i}\right)\left(\alpha_{11}+\alpha_{12} z^{k+1}\right)}, \\
& B_{n}^{n-k+1}=-\frac{\alpha_{10} \alpha_{12} y z^{k-1} z_{n-k}}{\left(\alpha_{11}+\alpha_{12} \prod_{i=0}^{k} z_{n-i}\right)\left(\alpha_{11}+\alpha_{12} z^{k+1}\right)}, \\
& B_{n}^{n-k}=-\frac{\alpha_{10} \alpha_{12} y z^{k}}{\left(\alpha_{11}+\alpha_{12} \prod_{i=0}^{k} z_{n-i}\right)\left(\alpha_{11}+\alpha_{12} z^{k+1}\right)}, \\
& C_{n}^{n}=-\frac{\alpha_{13} \alpha_{15} z \prod_{i=1}^{k} x_{n-i}}{\left(\alpha_{14}+\alpha_{15} \prod_{i=0}^{k} x_{n-i}\right)\left(\alpha_{14}+\alpha_{15} x^{k+1}\right)}, \\
& C_{n}^{n-1}=-\frac{\alpha_{13} \alpha_{15} z x \prod_{i=2}^{k} x_{n-i}}{\left(\alpha_{14}+\alpha_{15} \prod_{i=0}^{k} x_{n-i}\right)\left(\alpha_{14}+\alpha_{15} x^{k+1}\right)}, \\
& C_{n}^{n-k+1}=-\frac{\alpha_{13} \alpha_{15} z x^{k-1} x_{n-k}}{\left(\alpha_{14}+\alpha_{15} \prod_{i=0}^{k} x_{n-i}\right)\left(\alpha_{14}+\alpha_{15} x^{k+1}\right)}, \\
& C_{n}^{n-k}=-\frac{\alpha_{13} \alpha_{15} z x^{k}}{\left(\alpha_{14}+\alpha_{15} \prod_{i=0}^{k} x_{n-i}\right)\left(\alpha_{14}+\alpha_{15} x^{k+1}\right)} \text {, } \\
& D_{n}^{n-k}=\frac{\alpha_{13}}{\alpha_{14}+\alpha_{15} \prod_{i=0}^{1} x_{n-k}}, \\
& E_{n}^{n-k}=\frac{\alpha_{16}}{\alpha_{17}+\alpha_{18} \prod_{i=0}^{k} y_{n-i}}, \\
& F_{n}^{n}=-\frac{\alpha_{16} \alpha_{18} x \prod_{i=1}^{k} y_{n-i}}{\left(\alpha_{17}+\alpha_{18} \prod_{i=0}^{k} y_{n-i}\right)\left(\alpha_{17}+\alpha_{18} y^{k+1}\right)}, \\
& F_{n}^{n-1}=-\frac{\alpha_{16} \alpha_{18} x y \prod_{i=2}^{k} y_{n-i}}{\left(\alpha_{17}+\alpha_{18} \prod_{i=0}^{k} y_{n-i}\right)\left(\alpha_{17}+\alpha_{18} y^{k+1}\right)}, \\
& F_{n}^{n-k+1}=-\frac{\alpha_{16} \alpha_{18} x y^{k-1} y_{n-k}}{\left(\alpha_{17}+\alpha_{18} \prod_{i=0}^{k} y_{n-i}\right)\left(\alpha_{17}+\alpha_{18} y^{k+1}\right)}, \\
& F_{n}^{n-k}=\frac{\alpha_{16} \alpha_{18} x y^{k}}{\left(\alpha_{17}+\alpha_{18} \prod_{i=0}^{k} y_{n-i}\right)\left(\alpha_{17}+\alpha_{18} y^{k+1}\right)} .
\end{aligned}
$$


From (69) and (70), we get

$$
\lim _{n \rightarrow \infty} A_{n}^{n-k}=\frac{\alpha_{10}}{\alpha_{11}+\alpha_{12} z^{k+1}}
$$

$\lim _{n \longrightarrow \infty} B_{n}^{n}=\lim _{n \longrightarrow \infty} B_{n}^{n-1}=\cdots=\lim _{n \longrightarrow \infty} B_{n}^{n-k+1}=\lim _{n \longrightarrow \infty} B_{n}^{n-k}=-\frac{\alpha_{10} \alpha_{12} y z^{k}}{\left(\alpha_{11}+\alpha_{12} z^{k+1}\right)^{2}}$,

$\lim _{n \longrightarrow \infty} C_{n}^{n}=\lim _{n \longrightarrow \infty} C_{n}^{n-1}=\cdots=\lim _{n \longrightarrow \infty} C_{n}^{n-k+1}=\lim _{n \longrightarrow \infty} C_{n}^{n-k}=-\frac{\alpha_{13} \alpha_{15} z x^{k}}{\left(\alpha_{14}+\alpha_{15} x^{k+1}\right)^{2}}$,

$$
\begin{aligned}
& \lim _{n \longrightarrow \infty} D_{n}^{n-k}=\frac{\alpha_{13}}{\alpha_{14}+\alpha_{15} x^{k+1}}, \\
& \lim _{n \longrightarrow \infty} E_{n}^{n-k}=\frac{\alpha_{16}}{\alpha_{17}+\alpha_{18} y^{k+1}},
\end{aligned}
$$

$\lim _{n \longrightarrow \infty} F_{n}^{n}=\lim _{n \longrightarrow \infty} F_{n}^{n-1}=\cdots=\lim _{n \longrightarrow \infty} F_{n}^{n-k+1}=\lim _{n \longrightarrow \infty} F_{n}^{n-k}=-\frac{\alpha_{16} \alpha_{18} x y^{k}}{\left(\alpha_{17}+\alpha_{18} y^{k+1}\right)^{2}}$
So the limiting system [23]:

$$
\widetilde{\varrho_{n+1}}=\tilde{K} \widetilde{\varrho_{n}}
$$

where $\widetilde{Q_{n}}$ is depicted in (62) and $\tilde{K}$ is same as $\left.J\right|_{\wedge}$ about $\wedge$. In particular about $P_{0}$, it becomes

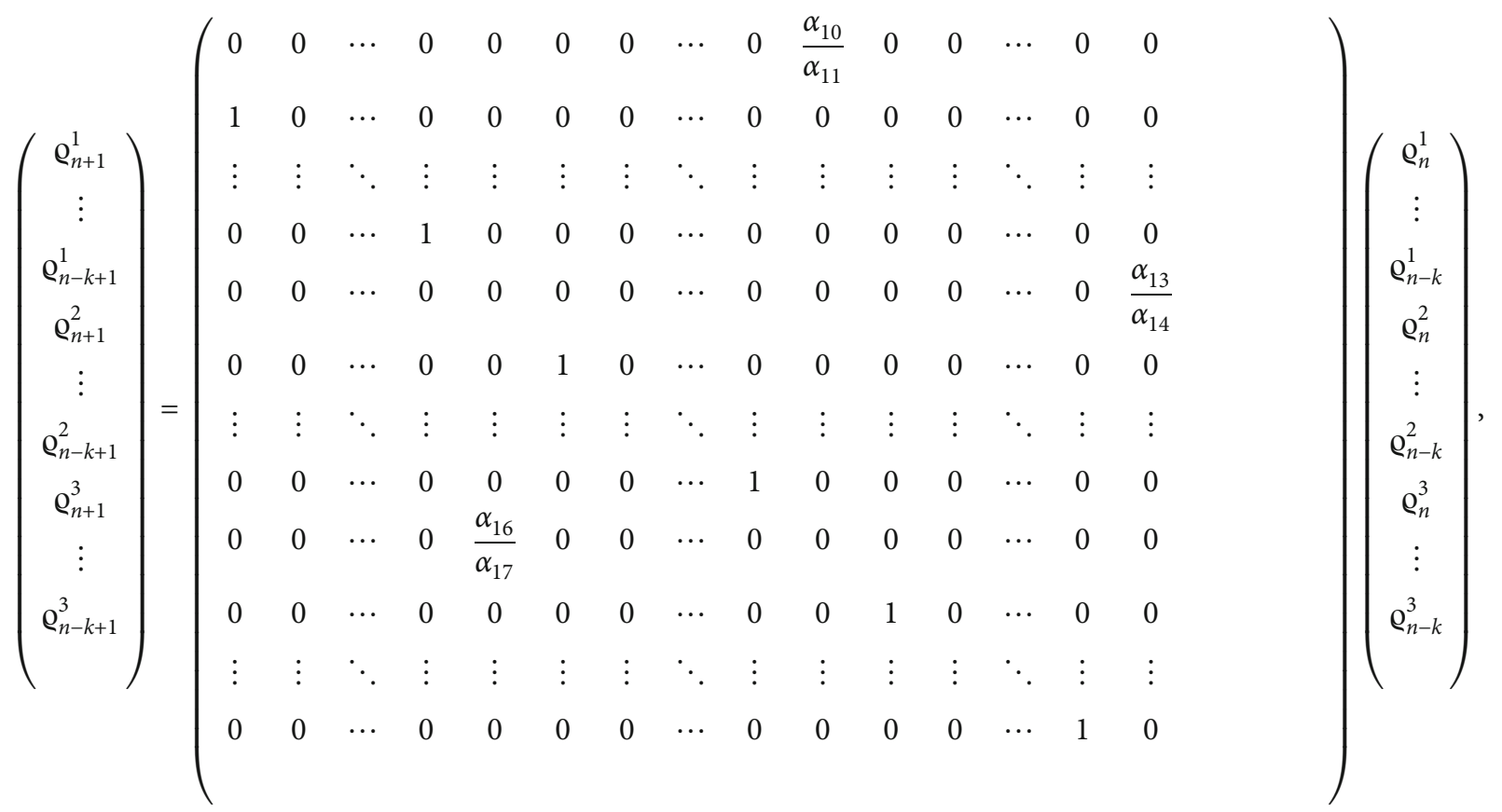

which is the same as the linearized system of (11) about $P_{0}$. Adopting a similar procedure, one can prove the rest of the results for discrete systems (12)-(14).

\section{Numerical Simulations}

We give some simulations in this section to verify not only the main finding but also show different behavior of discrete systems (11)-(14). For the systems under consideration, these numerical simulations provide time plots for $x_{n}, y_{n}$, $z_{n}$, as well as a global attractor. The following scenarios should be considered in this regard:
Example 1. Figure 1 indicates the behavior of discrete system (11) about $P_{0}$ if $k=2, \alpha_{\ell}(\ell=1,2, \cdots, 9)$, respectively, are 4,5 , $3,25,26,1,32,33$, and 4 and initial values $x_{-\ell}, \ell_{-\ell}, z_{-\ell}$ $(\ell=2,1,0)$, respectively, are $0.7,0.9,0.4,1.1,0.9,0.4,0.9$, 0.9 , and 0.8 . Moreover, Figures $1(\mathrm{a})-1(\mathrm{c})$ show that $P_{0}$ of discrete system (11) is a sink, and its attractor is shown in Figure 1(d).

Example 2. Figure 2 indicates the behavior of discrete system (11) about $P_{0}$ if $k=2, \alpha_{\ell}(\ell=1,2, \cdots, 9)$, respectively, are 6,5 , $0.1,25,22,0.3,32,30$, and 0.4 and initial values $x_{-\ell}, \ell_{-\ell}$, and 


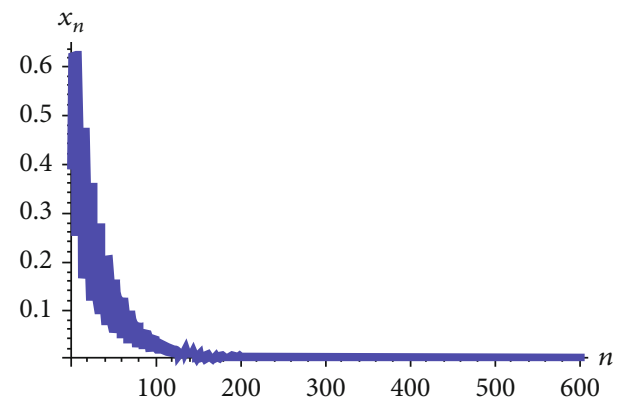

(a)

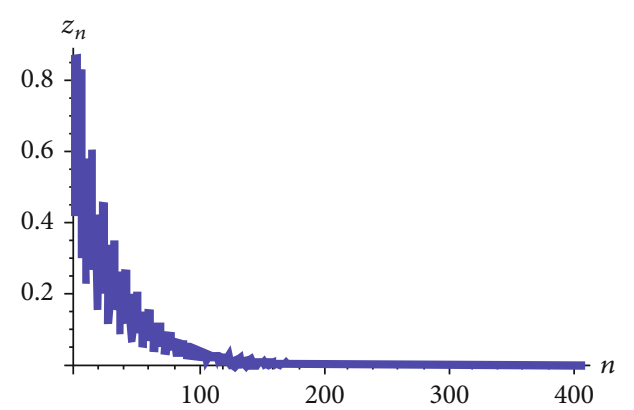

(c)

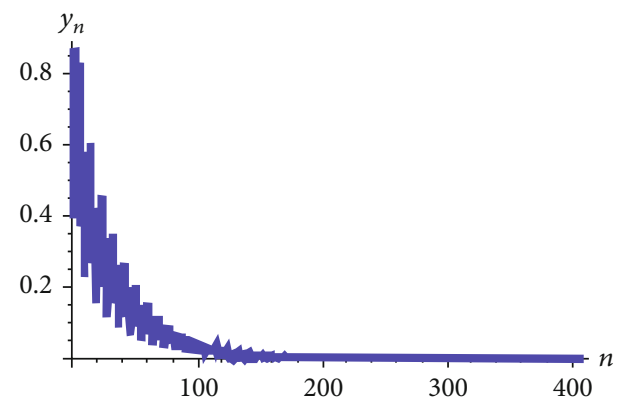

(b)

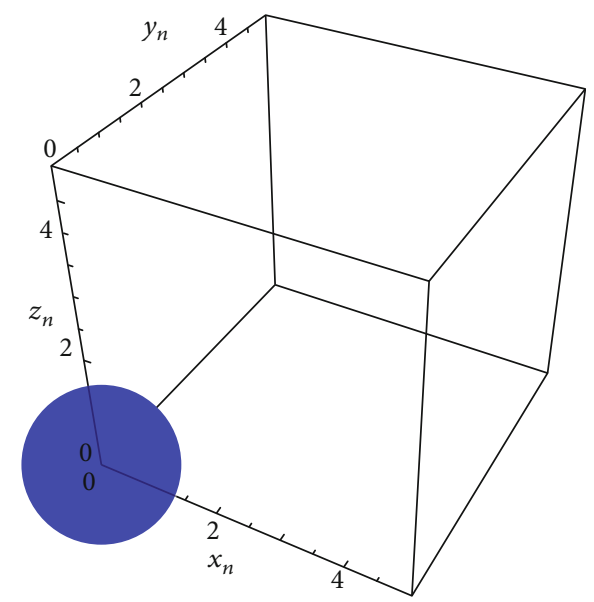

(d)

Figure 1: Dynamical characteristics of (11).

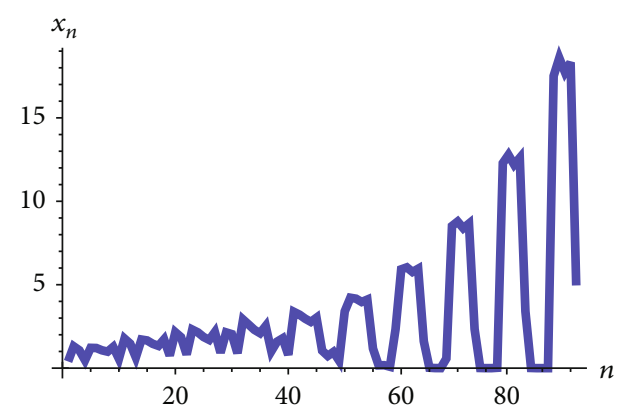

(a)

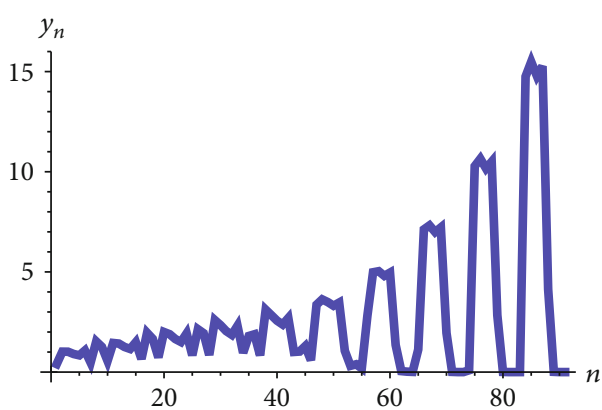

(b)

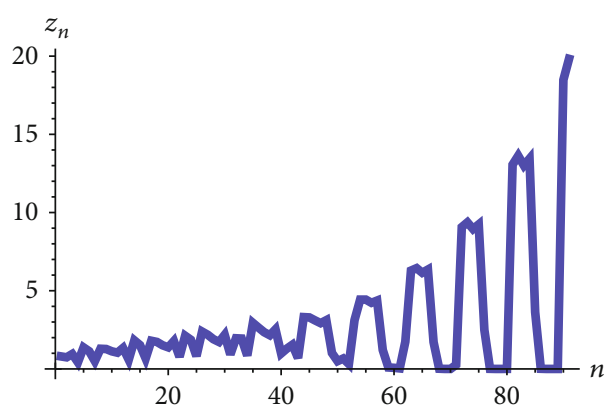

(c)

Figure 2: Dynamical characteristics of (11). 


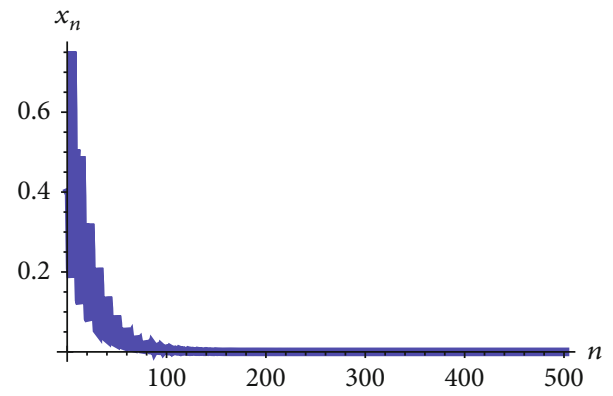

(a)

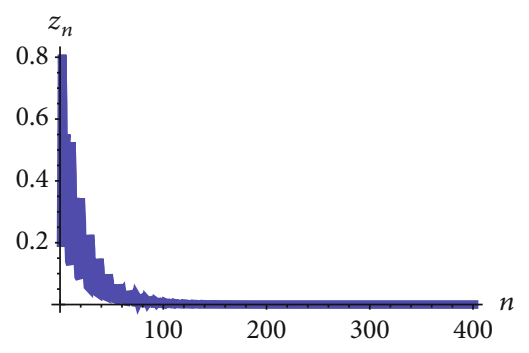

(c)

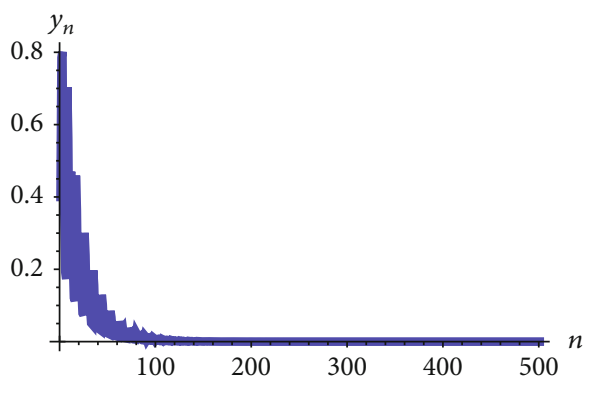

(b)

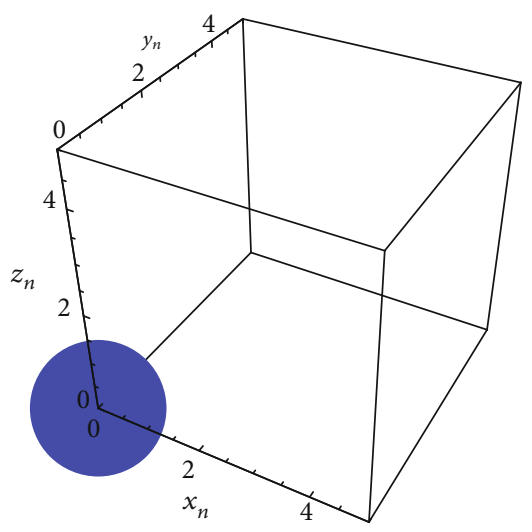

(d)

Figure 3: Dynamical characteristics of (12).

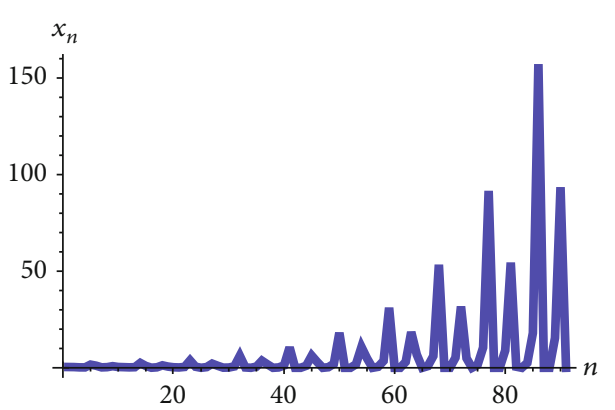

(a)

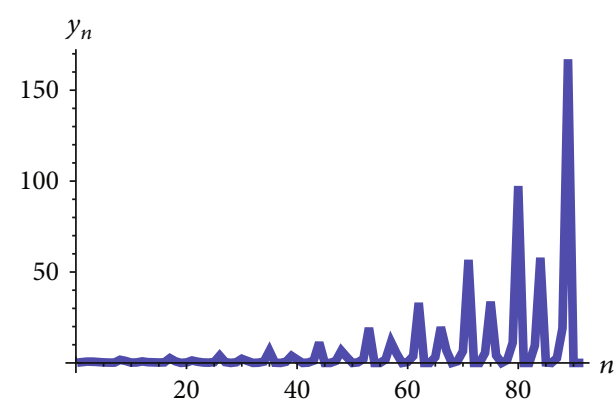

(b)

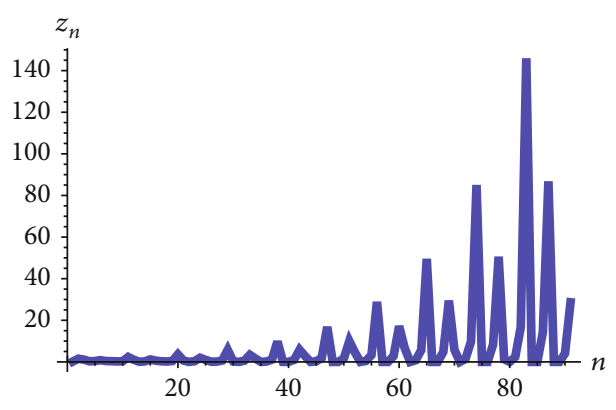

(c)

FIGURe 4: Dynamical characteristics of (12).

$z_{-\ell}(\ell=2,1,0)$, respectively, are $0.7,0.9,0.4,1.1,0.9,0.4,0.9$, 0.9 , and 0.8. Moreover, Figures 2(a)-2(c) show that $P_{0}$ of discrete system (11) is unstable.
Example 3. Figure 3 indicates the behavior of discrete system (12) about $P_{0}$ if $k=2, \alpha_{\ell}(\ell=1,2, \cdots, 9)$, respectively, are 14 , $15,3,15,16,3,3,4$, and 4 and $x_{-\ell}, \ell_{-\ell}$, and $z_{-\ell}(\ell=2,1,0)$, 


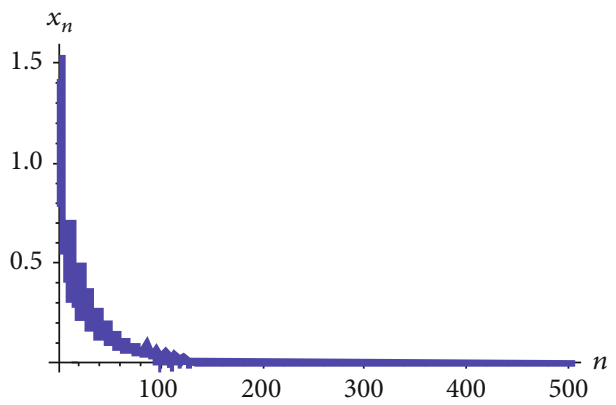

(a)

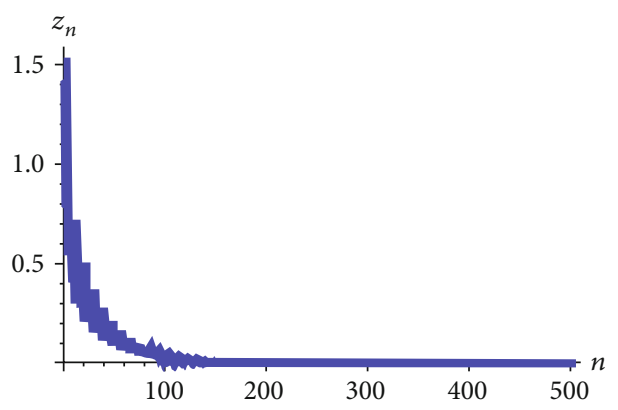

(c)

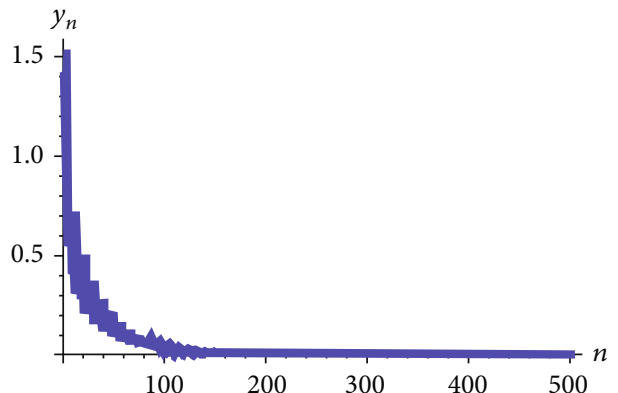

(b)

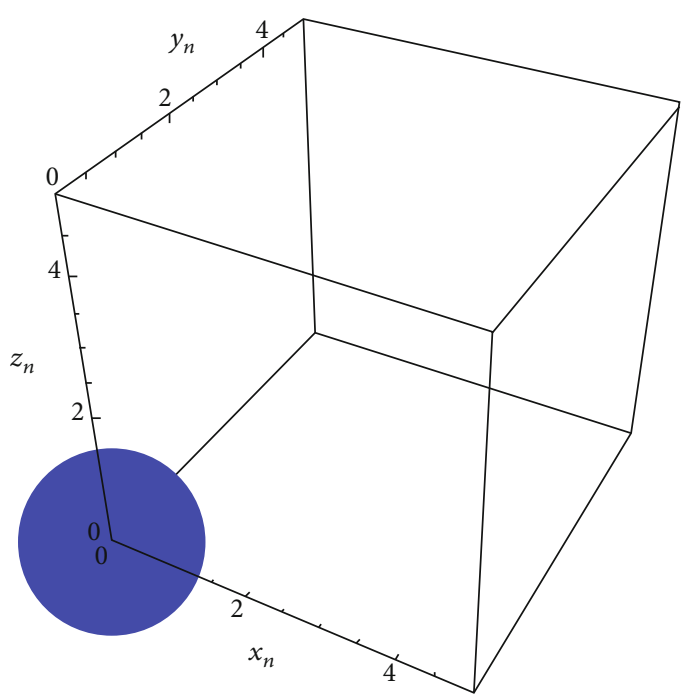

(d)

FIgURE 5: Dynamical characteristics of (13).

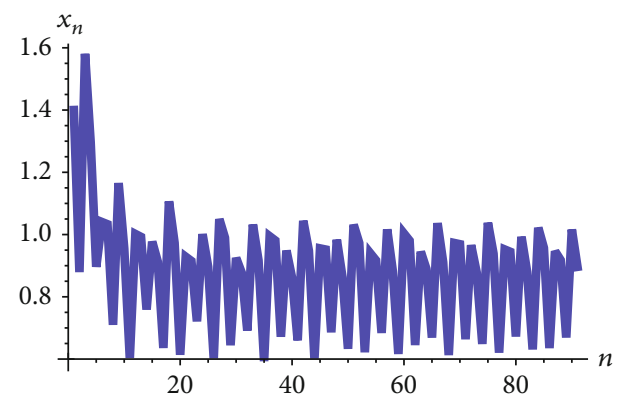

(a)

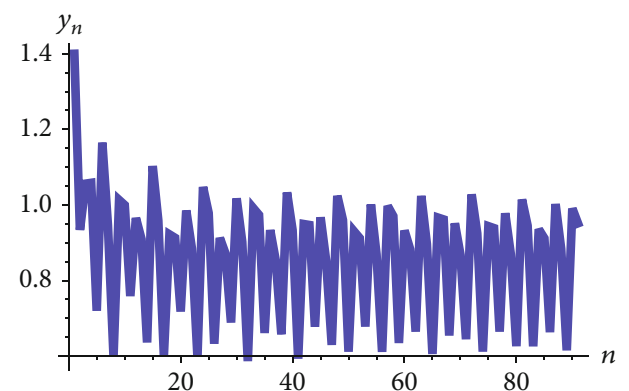

(b)

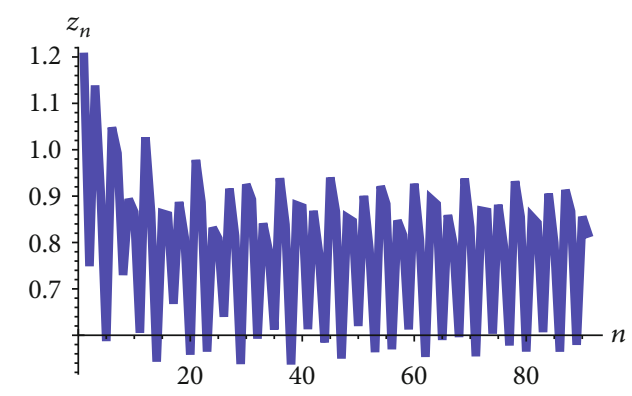

(c)

Figure 6: Dynamical characteristics of (13). 


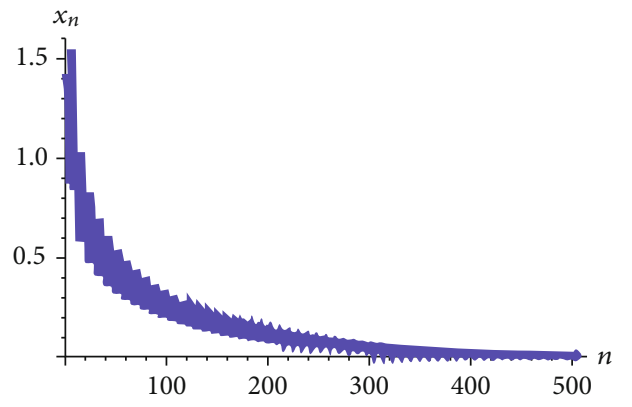

(a)

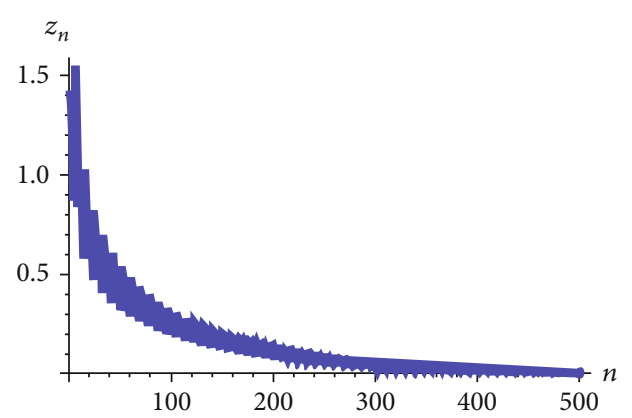

(c)

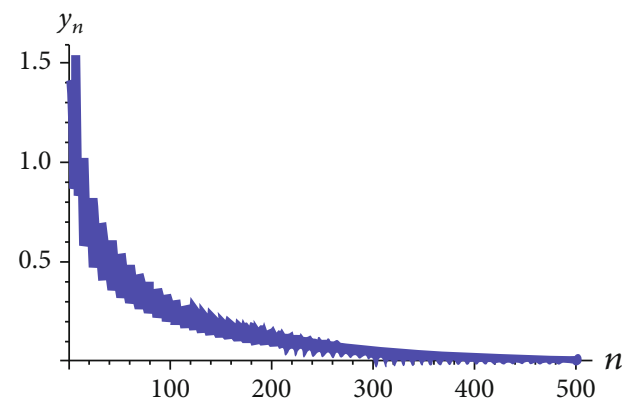

(b)

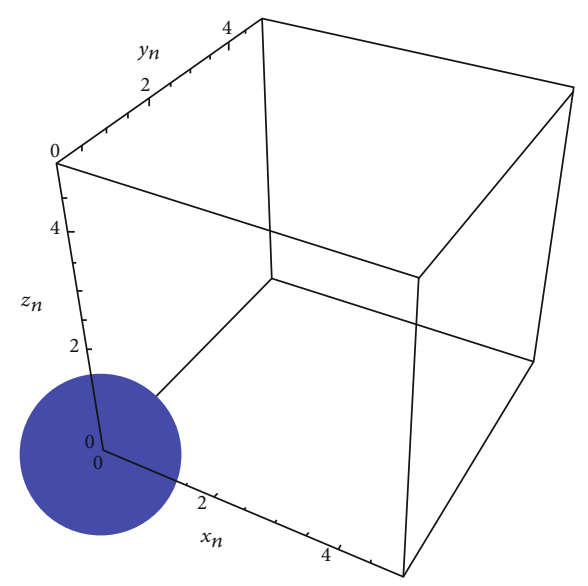

(d)

Figure 7: Dynamical characteristics of (14).

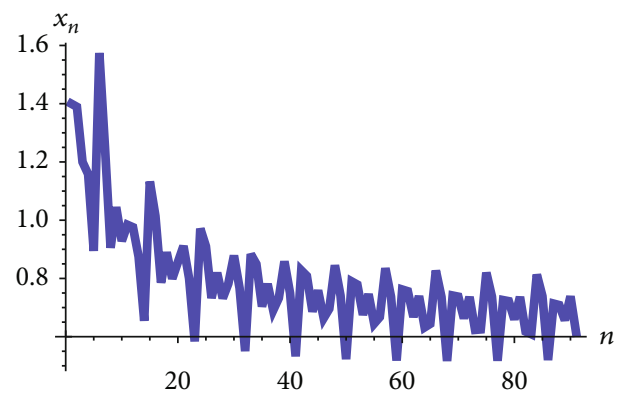

(a)

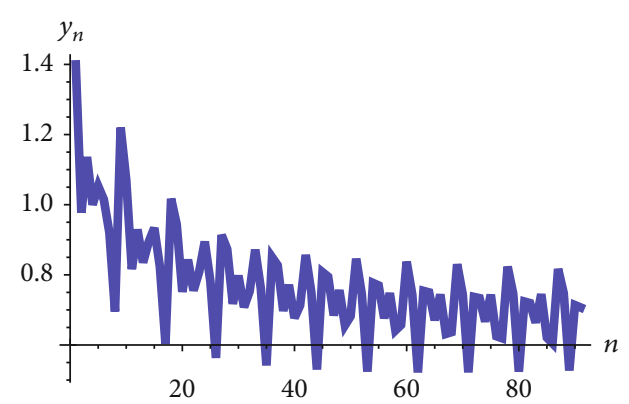

(b)

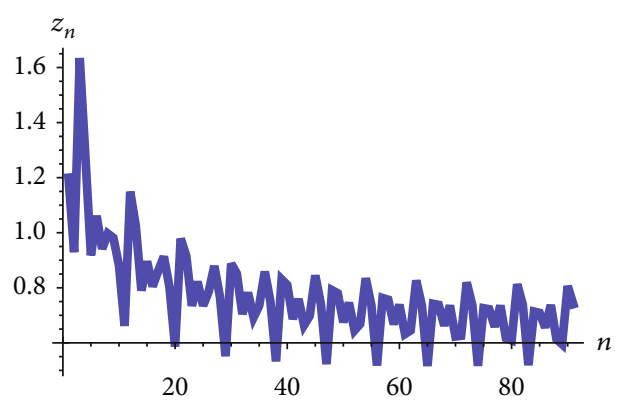

(c)

FIgure 8: Dynamical characteristics of (14). 
respectively, are $0.9,0.9,0.4,1.1,0.9,0.4,0.45,0.23$, and 0.2 . Moreover, Figures 3(a)-3(c) show that $P_{0}$ of discrete system (12) is a sink, and its attractor is shown in Figure 3(d).

Example 4. Figure 4 indicates the behavior of discrete system (12) about $P_{0}$ if $k=2, \alpha_{\ell}(\ell=1,2, \cdots, 9)$, respectively, are 14 , $13,13,17,16,13,6,4$, and 14 and $x_{-\ell}, \ell_{-\ell}$, and $z_{-\ell}$ $(\ell=2,1,0)$, respectively, are $0.9,0.9,0.4,1.1,0.9,0.4,0.45$, 0.23 , and 0.2. Moreover, Figures $4(\mathrm{a})-4(\mathrm{c})$ show that $P_{0}$ of discrete system (12) is unstable.

Example 5. Figure 5 indicates the behavior of discrete system (13) about $P_{0}$ if $k=2, \alpha_{\ell}(\ell=1,2, \cdots, 9)$, respectively, are 24 , $25,3,5,6,3,22,23$, and 8 and $x_{-\ell}, \ell_{-\ell}$, and $z_{-\ell}(\ell=2,1,0)$, respectively, are $1.9,1.9,1.4,1.1,1.9,1.4,1.45,1.23$, and 1.2. Moreover, Figures 5(a)-5(c) show that $P_{0}$ of discrete system (13) is a sink, and its attractor is shown in Figure 5(d).

Example 6. Figure 6 indicates the behavior of discrete system (13) about $P_{0}$ if $k=2, \alpha_{\ell}(\ell=1,2, \cdots, 9)$, respectively, are 27, $25,3,8,6,3,25,23$, and 8 and $x_{-\ell}, \ell_{-\ell}$, and $z_{-\ell}(\ell=2,1,0)$, respectively, are $1.9,1.9,1.4,1.1,1.9,1.4,1.45,1.23$, and 1.2. Moreover, Figures 6(a)-6(c) show that $P_{0}$ of discrete system (13) is unstable.

Example 7. Figure 7 indicates the behavior of discrete system (14) about $P_{0}$ if $k=2, \alpha_{\ell}(\ell=1,2, \cdots, 9)$, respectively, are 124, $125,3,5,6,3,122,123$, and 8 and $x_{-\ell}, \ell_{-\ell}$, and $z_{-\ell}(\ell=2,1,0)$ , respectively, are 1.9, 1.9, 1.4, 1.1, 1.9, 1.4, 1.45, 1.23, and 1.2. Moreover, Figures 7(a)-7(c) show that $P_{0}$ of discrete system (14) is a sink, and its attractor is shown in Figure 7(d).

Example 8. Figure 8 indicates the behavior of discrete system (14) about $P_{0}$ if $k=2, \alpha_{\ell}(\ell=1,2, \cdots, 9)$, respectively, are 124, $123,3,15,14,3,122,121$, and 8 and $x_{-\ell}, \ell_{-\ell}$, and $z_{-\ell}$ $(\ell=2,1,0)$, respectively, are $1.9,1.9,1.4,1.1,1.9,1.4,1.45$, 1.23 , and 1.2. Moreover, Figures $8(\mathrm{a})-8(\mathrm{c})$ show that $P_{0}$ of discrete system (14) is unstable.

\section{Conclusion and Future Work}

In this paper, we explored global dynamical characteristics, boundedness, and convergence rate of certain higher-order discrete systems of difference equations which is a natural extension of [21,22]. We have proved that trivial fixed point $P_{0}$ of discrete systems (11)-(14) is globally stable if, respectively, parametric conditions (i) $\alpha_{10} / \alpha_{11}<1, \alpha_{13} / \alpha_{14}<1$, and $\alpha_{16} / \alpha_{17}<1$; (ii) $\alpha_{19} / \alpha_{20}<1, \alpha_{22} / \alpha_{23}<1$, and $\alpha_{25} / \alpha_{26}<1$; (iii) $\alpha_{28} / \alpha_{29}<1, \alpha_{31} / \alpha_{32}<1$, and $\alpha_{34} / \alpha_{35}<1$; and (iv) $\alpha_{37} /$ $\alpha_{38}<1, \alpha_{40} / \alpha_{41}<1$, and $\alpha_{43} / \alpha_{44}<1$ hold. Further, we have proved that every positive solution of discrete systems (11)-(14) is bounded if (i) $\alpha_{10} / \alpha_{11}<1, \alpha_{13} / \alpha_{14}<1$, and $\alpha_{16} /$ $\alpha_{17}<1$; (ii) $\alpha_{19} / \alpha_{20}<1, \alpha_{22} / \alpha_{23}<1$, and $\alpha_{25} / \alpha_{26}<1$; (iii) $\alpha_{28}$ $/ \alpha_{29}<1, \alpha_{31} / \alpha_{32}<1$, and $\alpha_{34} / \alpha_{35}<1$; and (iv) $\alpha_{37} / \alpha_{38}<1$, $\alpha_{40} / \alpha_{41}<1$, and $\alpha_{43} / \alpha_{44}<1$ hold. It is also examined that positive solution of discrete systems (11)-(14) converges to $P_{0}$. Finally, numerical verification of theoretical results is performed. Closed-form solution and calculation of forbid- den set for the discrete systems (11)-(14) are our next aim to study.

\section{Data Availability}

All the data utilized in this article have been included, and the sources from where they were adopted were cited accordingly.

\section{Conflicts of Interest}

The authors declare that they have no conflicts of interest.

\section{References}

[1] H. Sedaghat, Nonlinear Difference Equations: Theory with Applications to Social Science Models, Springer, Netherlands, 2003.

[2] V. L. Kocic and G. Ladas, Global Behavior of Nonlinear Difference Equations of Higher-Order with Applications, Kluwer Academic, Dordrecht, 1993.

[3] B. Oğul, D. Şimsek, A. S. Kurbanli, and H. Öğünmez, "Dynamical behavior of rational difference equation $x_{n+1}=x_{n-15} / \pm 1$ $\pm x_{n-3} x_{n-7} x_{n-11} x_{n-15}$," Differential Equations and Dynamical Systems, pp. 1-16, 2021.

[4] M. R. S. Kulenović, G. Ladas, and N. P. Prokup, "A rational difference equation," Computers and Mathematics with Applications, vol. 41, no. 5-6, pp. 671-678, 2001.

[5] Q. Zhang, W. Zhang, Y. Shao, and J. Liu, "On the system of high order rational difference equations," International Scholarly Research Notices, vol. 2014, Article ID 760502, 5 pages, 2014.

[6] S. Kalabusisić, M. R. S. Kulenović, and E. Pilav, "Global dynamics of a competitive system of rational difference equations in the plane," Advances in Difference Equations, vol. 2009, Article ID 132802, 30 pages, 2009.

[7] S. Kalabusisić, M. R. S. Kulenović, and E. Pilav, "Dynamics of a two-dimensional system of rational difference equations of Leslie-Gower type," Advances in Difference Equations, vol. 2011, no. 1, 2011.

[8] S. Kalabušić, M. R. S. Kulenović, and E. Pilav, "Multiple attractors for a competitive system of rational difference equations in the plane," Abstract and Applied Analysis, vol. 2011, Article ID 295308, 35 pages, 2011.

[9] M. Garić-Demirović, M. R. S. Kulenović, and M. Nurkanović, "Global behavior of four competitive rational systems of difference equations in the plane," Discrete Dynamics in Nature and Society, vol. 2009, Article ID 153058, 34 pages, 2009.

[10] E. M. Elsayed, "Solutions of rational difference systems of order two," Mathematical and Computer Modelling, vol. 55, no. 3-4, pp. 378-384, 2012.

[11] E. M. Elsayed, "Behavior and expression of the solutions of some rational difference equations," Journal of Computational Analysis and Applications, vol. 15, no. 1, pp. 73-81, 2013.

[12] A. Q. Khan and M. N. Qureshi, "Global dynamics of a competitive system of rational difference equations," Mathematical Methods in the Applied Sciences, vol. 38, no. 18, pp. 47864796, 2015.

[13] R. DeVault, G. Ladas, and S. W. Schultz, "On the recursive sequence $x_{n+1}=\left(A / x_{n}\right)+\left(1 / x_{n-2}\right)$," Proceedings of the 
American Mathematical Society, vol. 126, no. 11, pp. 32573261, 1998.

[14] R. M. Abu-Saris and R. DeVault, "Global stability of $x_{n+1}=A$ $+\left(x_{n} / x_{n-k}\right)$," Applied Mathematics Letters, vol. 16, no. 2, pp. 173-178, 2003.

[15] S. Elaydi, An Introduction to Difference Equations, SpringerVerlag, New York, 2005.

[16] E. A. Grove and G. Ladas, Periodicities in Nonlinear Difference Equations, Chapman and Hall/CRC, Boca Raton, 2004.

[17] M. R. S. Kulenović and G. Ladas, Dynamics of Second-Order Rational Difference Equations, Chapman and Hall/CRC, London, 2002.

[18] E. Camouzis and G. Ladas, Dynamics of Third-Order Rational Difference Equations: With Open Problems and Conjectures, Chapman and Hall/HRC, Boca Raton, 2007.

[19] Q. Wang, Q. Zhang, and Q. Li, "Dynamics of a higher-order system of difference equations," Discrete Dynamics in Nature and Society, vol. 2017, Article ID 4312640, 9 pages, 2017.

[20] A. Khaliq, H. S. Alayachi, M. S. M. Noorani, and A. Q. Khan, "On stability analysis of higher-order rational difference equation," Discrete Dynamics in Nature and Society, vol. 2020, Article ID 3094185, 10 pages, 2020.

[21] I. Bajo and E. Liz, "Global behaviour of a second-order nonlinear difference equation," Journal of Difference Equations and Applications, vol. 17, no. 10, pp. 1471-1486, 2011.

[22] Q. Zhang, L. Yang, and J. Liu, "Dynamics of a system of rational third order difference equation," Advances in Difference Equations, vol. 2012, Article ID 136, 2012.

[23] M. Pituk, "More on Poincaré's and Perron's theorems for difference equations*," Journal of Difference Equations and Applications, vol. 8, no. 3, pp. 201-216, 2002. 